\title{
TU/e EmonOWEN

\section{Fluctuations of the partition function in the generalized random energy model with external field}

Citation for published version (APA):

Bovier, A., \& Klymovskiy, A. (2008). Fluctuations of the partition function in the generalized random energy model with external field. Journal of Mathematical Physics, 49(12), 125202-1/27.

https://doi.org/10.1063/1.2962982

DOI:

10.1063/1.2962982

Document status and date:

Published: 01/01/2008

\section{Document Version:}

Publisher's PDF, also known as Version of Record (includes final page, issue and volume numbers)

\section{Please check the document version of this publication:}

- A submitted manuscript is the version of the article upon submission and before peer-review. There can be important differences between the submitted version and the official published version of record. People interested in the research are advised to contact the author for the final version of the publication, or visit the $\mathrm{DOI}$ to the publisher's website.

- The final author version and the galley proof are versions of the publication after peer review.

- The final published version features the final layout of the paper including the volume, issue and page numbers.

Link to publication

\section{General rights}

Copyright and moral rights for the publications made accessible in the public portal are retained by the authors and/or other copyright owners and it is a condition of accessing publications that users recognise and abide by the legal requirements associated with these rights.

- Users may download and print one copy of any publication from the public portal for the purpose of private study or research.

- You may not further distribute the material or use it for any profit-making activity or commercial gain

- You may freely distribute the URL identifying the publication in the public portal.

If the publication is distributed under the terms of Article 25fa of the Dutch Copyright Act, indicated by the "Taverne" license above, please follow below link for the End User Agreement:

www.tue.nl/taverne

Take down policy

If you believe that this document breaches copyright please contact us at:

openaccess@tue.nl

providing details and we will investigate your claim. 


\title{
Fluctuations of the partition function in the generalized random energy model with external field
}

\author{
Anton Bovier ${ }^{1,2, a)}$ and Anton Klimovsky ${ }^{2, b)}$ \\ ${ }^{1}$ Weierstraß-Institut für Angewandte Analysis und Stochastik, Mohrenstraße 39, \\ 10117 Berlin, Germany \\ ${ }^{2}$ Institut für Mathematik, Technische Universität Berlin, Straße des 17 Juni 136, \\ 10623 Berlin, Germany
}

(Received 12 May 2008; accepted 26 June 2008; published online 3 December 2008)

\begin{abstract}
We study Derrida's generalized random energy model (GREM) in the presence of uniform external field. We compute the fluctuations of the ground state and of the partition function in the thermodynamic limit for all admissible values of parameters. We find that the fluctuations are described by a hierarchical structure which is obtained by a certain coarse graining of the initial hierarchical structure of the GREM with external field. We provide an explicit formula for the free energy of the model. We also derive some large deviation results providing an expression for the free energy in a class of models with Gaussian Hamiltonians and external field. Finally, we prove that the coarse-grained parts of the system emerging in the thermodynamic limit tend to have a certain optimal magnetization, as prescribed by the strength of the external field and by parameters of the GREM. (0) 2008 American Institute of Physics. [DOI: 10.1063/1.2962982]
\end{abstract}

\section{INTRODUCTION}

Despite the recent substantial progress due to Guerra, ${ }^{1}$ Aizenman et al. ${ }^{2,3}$ and Talagrand ${ }^{4}$ in establishing rigorously the Parisi formula for the free energy of the celebrated SherringtonKirkpatrick (SK) model, understanding of the corresponding limiting Gibbs measure is still very limited.

Due to the above mentioned works, it is now rigorously known that the generalized random energy model (GREM) introduced by Derrida ${ }^{5}$ is closely related to the SK model at the level of free energy, see, e.g., Bovier ${ }^{6}$ Sec. 11.3. Recently Bovier and Kurkova ${ }^{7-9}$ have performed a detailed study of the geometry of the Gibbs measure for the GREM. This confirmed the predicted in the theoretical physics literature hierarchical decomposition of the Gibbs measure in rigorous terms.

As pointed out by Bovier and Kurkova ${ }^{7}$ (see also Ben Arous et al. ${ }^{10}$ ), the GREM-like models may represent an independent interest in various applied contexts, where correlated heavy-tailed inputs play an important role, e.g., in risk modeling.

One of the key steps in the results of Bovier and Kurkova ${ }^{7}$ is the identification of the fluctuations of the GREM partition function in the thermodynamic limit with Ruelle's probability cascades. In this paper we also perform this step and study the effect of external field on the fluctuations (i.e., the weak limit laws) of the partition function of the GREM in the thermodynamic limit. We find that the main difference introduced by the presence of external field, comparing to the system without external field, is that the coarse graining mechanism should be altered. This change reflects the fact that the coarse-grained parts of the system tend to have a certain optimal magnetization as prescribed by the strength of the external field and by parameters of the GREM. We use the general line of reasoning suggested by Bovier and Kurkova, ${ }^{7}$ i.e., we consider the point

\footnotetext{
${ }^{a)}$ Electronic mail: bovier@wias-berlin.de.

${ }^{\text {b) }}$ Electronic mail: klimovsk@math.tu-berlin.de.
} 
processes generated by the scaling limits of the GREM Hamiltonian. We streamline the proof of the weak convergence of these point processes to the corresponding Poisson point process by using the Laplace transform.

\section{A. Organization of the paper}

In the following subsections of the Introduction we define the model of interest and formulate our main results on the fluctuations of the partition function of the random energy model (REM) and GREM with external field and also on their limiting free energy (Theorems 1.1-1.4). Their proofs are provided in the subsequent sections. Section II is devoted to the large deviation results providing an expression for the free energy for a class of models with Gaussian Hamiltonians and external field (Theorem 2.1). In Sec. III we resort to more refined analysis and perform the calculations of the fluctuations of the ground state and of the partition function in the REM with external field in the thermodynamic limit. Section VI contains the proofs of the results on the fluctuations of the ground state and of the partition function for the GREM with external field.

\section{B. Definition of the model}

Derrida's GREM was proposed as a mean-field spin-glass model with a Gaussian Hamiltonian and hierarchical correlation structure. In this paper, we consider the GREM with uniform external (magnetic) field. In contrast to the work of Derrida and Gardner, ${ }^{11}$ we consider here the model with the external field which depends linearly on the total magnetization (i.e., the uniform magnetic field). Derrida and Gardner ${ }^{11}$ considered the "lexicographic" external field which is particularly well adapted to the natural lexicographic distance generated by the GREM Hamiltonian.

Given $N \in \mathbb{N}$, consider the standard discrete hypercube $\Sigma_{N} \equiv\{-1 ; 1\}^{N}$. It will play the role of the index set. Define the (normalized) lexicographic overlap between the configurations $\sigma^{(1)}, \sigma^{(2)} \in \Sigma_{N}$ as

$$
q_{L}\left(\sigma^{(1)}, \sigma^{(2)}\right) \equiv \begin{cases}0, & \sigma_{1}^{(1)} \neq \sigma_{1}^{(2)} \\ \frac{1}{N} \max \left\{k \in[1 ; N] \cap \mathbb{N}:\left[\sigma^{(1)}\right]_{k}=\left[\sigma^{(2)}\right]_{k}\right\}, & \text { otherwise. }\end{cases}
$$

We equip the index set with the lexicographic distance defined as

$$
d_{L}\left(\sigma^{(1)}, \sigma^{(2)}\right) \equiv 1-q_{L}\left(\sigma^{(1)}, \sigma^{(2)}\right) .
$$

This distance is obviously an ultrametric, that is, for all $\sigma^{(1)}, \sigma^{(2)}, \sigma^{(3)} \in \Sigma_{N}$, we have

$$
d_{L}\left(\sigma^{(1)}, \sigma^{(3)}\right) \leq \max \left\{d_{L}\left(\sigma^{(1)}, \sigma^{(2)}\right), d_{L}\left(\sigma^{(2)}, \sigma^{(3)}\right)\right\} .
$$

Throughout the paper, we assume that we are given a large enough probability space $(\Omega, \mathcal{F}, \mathbb{P})$ such that all random variables under consideration are defined on it. Without further notice, we shall assume that all Gaussian random variables (vectors and processes) are centered.

Let $\operatorname{GREM}_{N} \equiv\left\{\operatorname{GREM}_{N}(\sigma)\right\}_{\sigma \in \Sigma_{N}}$ be the Gaussian random process on the discrete hypercube $\Sigma_{N}$ with the covariance of the following form:

$$
\mathbb{E}\left[\operatorname{GREM}_{N}\left(\sigma^{(1)}\right) \operatorname{GREM}_{N}\left(\sigma^{(2)}\right)\right]=\rho\left(q_{L}\left(\sigma^{(1)}, \sigma^{(2)}\right)\right),
$$

where $\rho:[0 ; 1] \rightarrow[0 ; 1]$ is the nondecreasing right-continuous function such that $\rho(0)=0$ and $\rho(1)=1$. Given $h \in \mathbb{R}_{+}$, consider the Gaussian process $X \equiv X_{N} \equiv\left\{X_{N}(h, \sigma)\right\}_{\sigma \in \Sigma_{N}}$ defined as

$$
X_{N}(h, \sigma) \equiv \operatorname{GREM}_{N}(\sigma)+\frac{h}{\sqrt{N}} \sum_{i=1}^{N} \sigma_{i}, \quad \sigma \in \Sigma_{N}
$$

The second summand in (1.3) is called the external field. The parameter $h$ represents the strength of external field. Denote the total magnetization by 


$$
m_{N}(\sigma) \equiv \frac{1}{N} \sum_{i=1}^{N} \sigma_{i}, \quad \sigma \in \Sigma_{N}
$$

The random process (1.3) induces the Gibbs measure $\mathcal{G}_{N}(\beta, h) \in \mathcal{M}_{1}\left(\Sigma_{N}\right)$ in the usual way,

$$
\mathcal{G}_{N}(\beta, h)(\{\sigma\}) \equiv \frac{1}{Z_{N}(\beta)} \exp \left[\beta \sqrt{N} X_{N}\left(\beta^{-1} h, \sigma\right)\right]
$$

where the normalizing constant $Z_{N}(\beta)$ is called the partition function $Z_{N}(\beta, h)$ and is given by the following sum of $2^{N}$ correlated exponentials:

$$
Z_{N}(\beta, h) \equiv \sum_{\sigma \in \Sigma_{N}} \exp \left[\beta \sqrt{N} X_{N}(h, \sigma)\right] .
$$

The real parameter $\beta>0$ is called the inverse temperature. The important quantities are the free energy defined as

$$
p_{N}(\beta, h) \equiv \frac{1}{N} \log Z_{N}(\beta, h),
$$

and the ground state energy

$$
M_{N}(h) \equiv N^{-1 / 2} \max _{\sigma \in \Sigma_{N}} X_{N}(h, \sigma) .
$$

In what follows, we shall think of $\beta$ and $h$ as fixed parameters. We shall occasionally lighten our notation by not indicating the dependence on these parameters explicitly.

In this paper we shall mainly be interested in the weak limit theorems (i.e., fluctuations) of the partition function (1.5) and of the ground state as $N \uparrow+\infty$. To be precise, the general results on Gaussian concentration of measure imply that (1.7) and (1.6) are self-averaging. By the fluctuations of the ground state, we mean the weak limiting behavior of the rescaled point process generated by the Gaussian process (1.3). This behavior is studied in Theorems 1.1 and 1.2 below. These theorems readily imply the formulas for the limiting free energy (1.6) and the ground state (1.7). A recent account of the mathematical results on the GREM without external field and, in particular, on the behavior of the limiting Gibbs measure can be found in the paper of Bovier and Kurkova. ${ }^{12}$ The GREM with external field was previously considered by Jana and Rao ${ }^{13}$ (see also $\mathrm{Jana}^{14}$ ), where its free energy was expressed in terms of a variational problem induced by an application of Varadhan's lemma. In this work, we apply very different methods to obtain precise control of the fluctuations of the partition function for the GREM with external field. As a simple consequence of these results, we also get a rather explicit ${ }^{1}$ formula for the limiting free energy in the GREM with external field (see Theorem 1.4).

\section{Main results}

In this paper, we shall consider the case of the piecewise constant function $\rho$ with a finite number of jumps. Consider the space of discrete order parameters,

$$
\mathcal{Q}_{n}^{\prime} \equiv\{q:[0 ; 1] \rightarrow[0 ; 1] \mid q(0)=0, q(1)=1,
$$

$q$ is nondecreasing, piecewise constant with $n$ jumps\}.

Recall the function $\rho$ from (1.2). Assume that $\rho \in \mathcal{Q}_{n}^{\prime}$. In what follows, we shall refer to $\rho$ as the discrete order parameter. In this case, it is possible to construct the process $\mathrm{GREM}_{N}$ as a finite sum of independent Gaussian processes. Assume that

${ }^{1}$ In contrast to Jana and Rao ${ }^{13}$ Theorem 5.1 and Jana ${ }^{14}$ Corollary 4.3.5, who stop at the level of variational problem. 


$$
\left.\rho(x)=\sum_{k=1}^{n} q_{k}\right]_{\left[x_{k} ; x_{k+1}\right)}(x),
$$

where

$$
\begin{aligned}
& 0 \equiv x_{0}<x_{1}<\cdots<x_{n}=1, \\
& 0 \equiv q_{0}<q_{1}<\cdots<q_{n}=1 .
\end{aligned}
$$

Let $\left\{a_{k}\right\}_{k=1}^{n} \subset \mathbb{R}$ be such that $a_{k}^{2}=q_{k}-q_{k-1}$. We assume that, for all $k \in[1 ; n] \cap \mathbb{N}$, we have $x_{k} N$ $\in \mathbb{N}^{2}$ and also $a_{k} \neq 0$. Denote $\Delta x_{l} \equiv x_{l}-x_{l-1}$.

Consider the family of independently and identically distributed standard Gaussian random variables,

$$
\left\{X\left(\sigma^{(1)}, \sigma^{(2)}, \ldots, \sigma^{(k)}\right) \mid k \in[1 ; n] \cap \mathbb{N}, \sigma^{(1)} \in \Sigma_{x_{1} N}, \ldots, \sigma^{(k)} \in \Sigma_{x_{k} N}\right\} .
$$

Using these ingredients, for $\sigma=\sigma^{(1)}\left\|\sigma^{(2)}\right\| \ldots \| \sigma^{(n)} \in \Sigma_{N}$, we have

$$
\operatorname{GREM}_{N}(\sigma) \sim \sum_{k=1}^{n} a_{k} X\left(\sigma^{(1)}, \sigma^{(2)}, \ldots, \sigma^{(k)}\right) .
$$

Equivalence (1.12) is easily verified by computing the covariance of the right hand side. The computation gives, for $\sigma, \tau \in \Sigma_{N}$,

$$
\operatorname{Cov}\left[\operatorname{GREM}_{N}(\sigma) \operatorname{GREM}_{N}(\tau)\right]=q_{N q_{L}(\sigma, \tau)} .
$$

\section{Limiting objects}

We now collect the objects which appear in weak limit theorems for the GREM partition function and for the ground states. We denote by $I:[-1 ; 1] \rightarrow \mathbb{R}_{+}$Cramér's entropy function, i.e.,

$$
I(t) \equiv \frac{1}{2}[(1-t) \log (1-t)+(1+t) \log (1+t)]
$$

Define

$$
\begin{gathered}
\mu(t) \equiv \sqrt{2(\log 2-I(t))}, \\
M(h) \equiv \max _{t \in[-1 ; 1]}(\mu(t)+h t) .
\end{gathered}
$$

Suppose that the maximum in (1.14) is attained at $t=t_{*}=t_{*}(h)$. [The maximum exists and is unique, since $\mu(t)+h t$ is strictly concave.] Consider the following two real sequences:

$$
\begin{gathered}
A_{N}(h) \equiv\left(\mu\left(t_{*}\right) \sqrt{N}\right)^{-1}, \\
B_{N}(h) \equiv M(h) \sqrt{N}+\frac{A_{N}(h)}{2} \log \left(\frac{A_{N}(h)^{2}\left(I^{\prime \prime}\left(t_{*}\right)+h\right)}{2 \pi\left(1-t_{*}^{2}\right)}\right) .
\end{gathered}
$$

Define the REM scaling function $u_{N, h}(x): \mathbb{R} \rightarrow \mathbb{R}$ as

\footnotetext{
${ }^{2}$ This condition is for notational simplicity. It means that we actually consider instead of $N$ the increasing sequence $\left\{N_{\alpha}\right\}_{\alpha \in \mathbb{N}} \subset \mathbb{N}$ such that $N_{\alpha} \uparrow+\infty$ as $\alpha \uparrow+\infty$, satisfying $N_{\alpha} x_{k} \in \mathbb{N}$, for all $\alpha \in \mathbb{N}$ and all $k \in[1 ; n] \cap \mathbb{N}$.
} 


$$
u_{N, h}(x) \equiv A_{N}(h) x+B_{N}(h) .
$$

Given $f: D \subset \mathbb{R} \rightarrow \mathbb{R}_{+}$, we denote by $\operatorname{PPP}(f(x) d x, x \in D)$ the Poisson point process with intensity $f$. We start from a basic limiting object. Assume that the point process $\mathcal{P}^{(1)}$ on $\mathbb{R}$ satisfies

$$
\mathcal{P}^{(1)} \sim \operatorname{PPP}(\exp (-x) d x, x \in \mathbb{R})
$$

and is independent of all random variables around. The point process (1.18) is the limiting object which appears in the REM.

Theorem 1.1: If $n=1$ (the REM case), then, using the above notations, we have

$$
\sum_{\sigma \in \Sigma_{N}} \delta_{u_{N, h}^{-1}\left(X_{N}(h, \sigma)\right)}^{\stackrel{w}{\longrightarrow}} \mathcal{P}^{(1)}
$$

where the convergence is the weak one of the random probability measures equipped with the vague topology.

Remark 1.1: It is easy to check that, for $h=0$, Theorem 1.1 reduces to the results of Bovier and Kurkova. ${ }^{15}$ Indeed, in this case $t_{*}(0)=0$, and, hence, $\mu(0)=M(0)=\sqrt{2 \log 2}$, which in turn implies that the scaling constants (1.15) and (1.16) coincide with that of the REM without external field.

To formulate the weak limit theorems for the GREM (i.e., for the case $n>1$ ), we need a limiting object which is a point process closely related to the Ruelle probability cascade $\left(\right.$ Ruelle $\left.^{16}\right)$. Define, for $j, k \in[1 ; n+1] \cap \mathbb{N}, j<k$, the "slopes" corresponding to the function $\rho$ in (1.2) as

$$
\theta_{j, k} \equiv \frac{q_{k}-q_{j-1}}{x_{k}-x_{j-1}}
$$

Define also the following $h$-dependent "modified slopes":

$$
\tilde{\theta}_{j, k}(h) \equiv \theta_{j, k} \mu\left(t_{*}\left(\theta_{j, k}^{-1 / 2} h\right)\right)^{-2} .
$$

Define the increasing sequence of indices $\left\{J_{l}(h)\right\}_{l=0}^{m(h)} \subset[0 ; n+1] \cap \mathbb{N}$ by the following algorithm. Start from $J_{0}(h) \equiv 0$ and define iteratively

$$
J_{l}(h) \equiv \min \left\{J \in\left[J_{l-1} ; n+1\right] \cap \mathbb{N}: \tilde{\theta}_{J_{l-1}, J}(h)>\tilde{\theta}_{J+1, k}(h), \text { for all } k>J\right\} .
$$

Note that $m(h) \leq n$. The subsequence of indices (1.21) induces the following coarse graining of the initial GREM:

$$
\begin{gathered}
\bar{q}_{l}(h) \equiv q_{J_{l}(h)}-q_{J_{l-1}(h)}, \\
\bar{x}_{l}(h) \equiv x_{J_{l}(h)}-x_{J_{l-1}(h)}, \\
\bar{\theta}_{l}(h) \equiv \theta_{J_{l-1}, J_{l}} .
\end{gathered}
$$

The parameters (1.22) induce the new order parameter $\rho^{(J(h))} \in \mathcal{Q}_{m}^{\prime}$ in the usual way,

$$
\rho^{(J(h))}(q) \equiv \sum_{l=1}^{m(h)} q_{J_{l}(h)} \mathbb{1}_{\left[x_{J_{l}(h)} ; x_{J_{l+1}}(h)\right.}(x) .
$$

Define the GREM scaling function $u_{N, \rho, h}: \mathbb{R} \rightarrow \mathbb{R}$ as 


$$
u_{N, \rho, h}(x) \equiv \sum_{l=1}^{m(h)}\left[\bar{q}_{l}(h)^{1 / 2} B_{\bar{x}_{l}(h) N}\left(\bar{\theta}_{l}(h)^{-1 / 2} h\right)\right]+N^{-1 / 2} x .
$$

Define the rescaled GREM process as

$$
\overline{\operatorname{GREM}}_{N}(h, \sigma) \equiv u_{N, \rho, h}^{-1}\left(\operatorname{GREM}_{N}(h, \sigma)\right) .
$$

Define the point process of the rescaled GREM energies $\mathcal{E}_{N}$ as

$$
\mathcal{E}_{N}(h) \equiv \sum_{\sigma \in \Sigma_{N}} \delta{\overline{\operatorname{GREM}_{N}(h, \sigma)}}
$$

Consider the following collection of independent point processes (which are also independent of all random objects introduced above):

$$
\left\{\mathcal{P}_{\alpha_{1}, \ldots, \alpha_{l-1}}^{(k)} \mid \alpha_{1}, \ldots, \alpha_{l-1} \in \mathbb{N} ; l \in[1 ; m] \cap \mathbb{N}\right\},
$$

such that

$$
\mathcal{P}_{\alpha_{1}, \ldots, \alpha_{k-1}}^{(k)} \sim \mathcal{P}^{(1)}
$$

Define the limiting GREM cascade point process $\mathcal{P}_{m}$ on $\mathbb{R}^{m}$ as follows:

$$
\mathcal{P}_{m} \equiv \sum_{\alpha \in \mathbb{N}^{m}} \delta_{\left(\mathcal{P}^{(1)}\left(\alpha_{1}\right), \mathcal{P}_{\alpha_{1}}^{(2)}\left(\alpha_{2}\right), \ldots, \mathcal{P}_{\alpha_{1}, \alpha_{2}, \ldots, \alpha_{m-1}}^{(m)}\left(\alpha_{m}\right)\right)}
$$

Consider the following constants:

$$
\bar{\gamma}_{l}(h) \equiv\left(\tilde{\theta}_{J_{l-1}, J_{l}}\right)^{1 / 2},
$$

and define the function $E_{h, f}: \mathbb{R}^{m} \rightarrow \mathrm{R}$ as

$$
E_{h, \rho}^{(m)}\left(e_{1}, \ldots, e_{m}\right) \equiv \bar{\gamma}_{1}(h) e_{1}+\cdots+\bar{\gamma}_{m}(h) e_{m} .
$$

Note that due to (1.21), the constants $\left\{\bar{\gamma}_{l}(h)\right\}_{l=1}^{m}$ form a decreasing sequence, i.e., for all $l$ $\in[1 ; m] \cap \mathbb{N}$, we have

$$
\bar{\gamma}_{l}(h)>\bar{\gamma}_{l+1}(h) .
$$

The cascade point process (1.26) is the limiting object which describes the fluctuations of the ground state in the GREM.

Theorem 1.2: We have

$$
\mathcal{E}_{N}(h) \underset{N \uparrow+\infty}{\stackrel{w}{\longrightarrow}} \int_{\mathbb{R}^{m}} \delta_{E_{h, \varrho}^{(m)}\left(e_{1}, \ldots, e_{m}\right)} \mathcal{P}_{m}\left(d e_{1}, \ldots, d e_{m}\right)
$$

and

$$
M_{N}(h) \underset{N \uparrow+\infty}{\rightarrow} \sum_{l=1}^{m(h)}\left[\left(\bar{q}_{l}(h) \bar{x}_{l}(h)\right)^{1 / 2} M\left(\bar{\theta}_{l}(h)^{-1 / 2} h\right)\right],
$$

almost surely and in $L^{1}$.

Theorem 1.2 allows for complete characterization of the limiting distribution of the GREM partition function. To formulate the result, we need the $\beta$-dependent threshold $l(\beta, h)$ $\in[0 ; m] \cap \mathbb{N}$ such that above it all coarse-grained levels $l>l(\beta, h)$ of the limiting GREM are in the "high temperature regime." Below this threshold the levels $l \leq l(\beta, h)$ are in the "frozen state." Given $\beta \in \mathbb{R}_{+}$, define 


$$
l(\beta, h) \equiv \max \left\{l \in[1 ; n] \cap \mathbb{N}: \beta \bar{\gamma}_{l}(h)>1\right\} .
$$

We set $l(\beta, h) \equiv 0$, if $\beta \bar{\gamma}_{1}(h) \leq 1$. The following gives full information about the limiting fluctuations of the partition function at all temperatures.

Theorem 1.3: We have

$$
\begin{aligned}
\exp [- & \left.\beta \sqrt{N} \sum_{l=1}^{l(\beta, h)}\left(\bar{q}_{l}(h)^{1 / 2} B_{\bar{x}_{l}(h) N}\left(\bar{\theta}_{l}^{-1 / 2} h\right)\right)\right] \exp \left[-N\left(\log 2+\log \operatorname{ch}\left(\beta h\left(1-x_{J_{l(\beta, h)}}\right)\right)\right.\right. \\
& \left.\left.+\frac{1}{2} \beta^{2}\left(1-q_{J_{l(\beta, h)}}\right)\right)\right] \operatorname{ch}^{2 / 3}\left(\beta h\left(1-x_{J_{l(\beta, h)}}\right)\right) Z_{N}(\beta, h) \\
& \stackrel{w}{\rightarrow} K(\beta, h, \rho) \int_{\mathbb{R}^{l(\beta, h)}} \exp \left[\beta E_{h, \rho}^{(l(\beta, h))}\left(e_{1}, \ldots, e_{l(\beta, h)}\right)\right] \mathcal{P}_{l(\beta, h)}\left(d e_{1}, \ldots, d e_{l(\beta, h)}\right),
\end{aligned}
$$

where the constant $K(\beta, h, \rho)$ depends on $\beta, h$, and $\rho$ only. Moreover, $K(\beta, h, \rho)=1$, if $\beta \gamma_{l(\beta, h)+1}$ $<1$ and $K(\beta, h, \rho) \in(0 ; 1)$, if $\beta \gamma_{l(\beta, h)+1}=1$.

The above theorem suggests that the increasing sequence of the constants $\left\{\beta_{l}\right.$ $\left.\equiv \bar{\gamma}^{-1}\right\}_{l=1}^{m(h)} \subset \mathbb{R}_{+}$can be thought of as the sequence of the inverse temperatures at which the phase transitions occur: at $\beta_{l}$ the corresponding coarse-grained level $l$ of the GREM with external field "freezes."

As a simple consequence of the fluctuation results of Theorem 1.3, we obtain the following formula for the limiting free energy of the GREM.

Theorem 1.4: We have

$$
\begin{aligned}
\lim _{N \uparrow+\infty} p_{N}(\beta, h)= & \beta \sum_{l=1}^{l(\beta, h)}\left[\left(\bar{x}_{l} \bar{q}_{l}\right)^{1 / 2} \mu\left(t_{*}\left(\bar{\theta}_{l}^{-1 / 2} h\right)\right)+h \bar{x}_{l} t_{*}\left(\bar{\theta}_{l}^{-1 / 2} h\right)\right]+\log 2+\log \operatorname{ch}\left(\beta h\left(1-x_{J_{l(\beta, h)}}\right)\right) \\
& +\frac{1}{2} \beta^{2}\left(1-q_{J_{l(\beta, h)}}\right),
\end{aligned}
$$

almost surely and in $L^{1}$.

Remark 1.2: For $h=0$, since $\mu\left(t_{*}(0)\right)=\mu(0)=\sqrt{2 \log 2}$ (see Remark 1.1), we have

$$
\tilde{\theta}_{j, k}(h)=\frac{\theta_{j, k}}{2 \log 2},
$$

which together with (1.21) recovers the coarse-graining algorithm of Bovier and Kurkova ${ }^{7}$ (1.15) for the GREM without external field.

\section{PARTIAL PARTITION FUNCTIONS, EXTERNAL FIELDS, AND OVERLAPS}

In this section, we propose a way to compute the free energy of disordered spin systems with external field using the restricted free energies of systems without external field. The computation involves a large deviations principle. For gauge invariant systems, we also show that the partition function of the system with external field induced by the total magnetization has the same distribution as the one induced by the overlap with fixed but arbitrary configuration. This section is based on the ideas of Derrida and Gardner. ${ }^{11}$

Fix $p \in \mathbb{N}$. Given some finite interaction $p$-hypergraph $\left(V_{N}, E_{N}^{(p)}\right)$, where $V_{N}=[1 ; n] \cap \mathbb{N}$ and $E_{N}^{(p)} \subset\left(V_{N}\right)^{p}$, define the $p$-spin interaction Hamiltonian as 


$$
X_{N}(\sigma) \equiv \sum_{i \in E_{N}^{(p)}} J_{i}^{(N, p)} \sigma_{i_{1}} \sigma_{i_{2}} \cdots \sigma_{i_{p}}, \quad \sigma \in \Sigma_{N},
$$

where $J^{(N, p)} \equiv\left\{J_{i}^{(N, p)}\right\}_{i \in E_{N}^{(p)}}$ is the collection of random variables having the symmetric joint distribution. That is, we assume that, for any $\varepsilon^{(1)}, \varepsilon^{(2)} \in\{-1 ;+1\}_{N}^{(p)}$ and any $t \in \mathbb{R}^{E_{N}^{(p)}}$,

$$
\mathbb{E}\left[\exp \left(i \sum_{r \in E_{N}^{(p)}} t_{r} \varepsilon_{r}^{(1)} J_{i}^{(N, p)}\right)\right]=\mathbb{E}\left[\exp \left(i \sum_{r \in E_{N}^{(p)}} t_{r} \varepsilon_{r}^{(2)} J_{i}^{(N, p)}\right)\right],
$$

where $i \in \mathrm{C}$ denotes the imaginary unit.

A particular important example of (2.1) is Derrida's $p$-spin Hamiltonian given by

$$
\operatorname{SK}_{N}^{(p)}(\sigma) \equiv N^{-p / 2} \sum_{i_{1}, \ldots, i_{p}=1}^{N} g_{i_{1}, \ldots, i_{p}} \sigma_{i_{1}} \sigma_{i_{2}} \cdots \sigma_{i_{p}},
$$

where $\left\{g_{i_{1}, \ldots, i_{p}}\right\}_{i_{1}, \ldots, i_{p}=1}^{N}$ is a collection of independently and identically distributed standard Gaussian random variables. Note that the condition (2.2) is obviously satisfied.

Given $\mu \in \Sigma_{N}$, define the corresponding gauge transformation $T_{\mu}: \Sigma_{N} \rightarrow \Sigma_{N}$ as

$$
T_{\mu}(\sigma)_{i}=\mu_{i} \sigma_{i}, \quad \sigma \in \Sigma_{N} .
$$

Note that the gauge transformation (2.3) is obviously an involution. We say that a $d$-variate random function $f: \Sigma_{N}^{d} \rightarrow \mathrm{R}$ is gauge invariant, if, for any $\mu \in \Sigma_{N}$ and any $\left(\sigma^{(1)}, \ldots, \sigma^{(d)}\right) \in \Sigma_{N}^{d}$,

$$
f\left(T_{\mu}\left(\sigma^{(1)}\right), \ldots, T_{\mu}\left(\sigma^{(d)}\right)\right) \sim f\left(\sigma^{(1)}, \ldots, \sigma^{(d)}\right),
$$

where $\sim$ denotes equality in distribution. Define the overlap between the configurations $\sigma, \sigma^{\prime}$ $\in \Sigma_{N}$ as

$$
R_{N}\left(\sigma, \sigma^{\prime}\right) \equiv \frac{1}{N} \sum_{i=1}^{N} \sigma_{i} \sigma_{i}^{\prime} .
$$

Note that the overlap (2.4) and the lexicographic overlap (1.1) are gauge invariant.

Given a bounded function $F_{N}: \Sigma_{N} \rightarrow \mathrm{R}$, define the partial partition function as

$$
Z_{N}^{(p)}\left(\beta, q, \varepsilon, X_{N}, F_{N}\right) \equiv \sum_{\sigma:\left|F_{N}(\sigma)-q\right| \leq \varepsilon} \exp \left(\beta \sqrt{N} X_{N}(\sigma)\right)
$$

Denote

$$
U_{N} \equiv F_{N}\left(\Sigma_{N}\right), \quad U \equiv \overline{\left(\bigcup_{N=1}^{\infty} U_{N}\right)} .
$$

[The bar in (2.6) denotes closure in the Euclidean topology.] Note that for the case $F_{N}=R_{N}$ we obviously have

$$
U_{N}=\left\{1-\frac{2 k}{N}: k \in[0 ; N] \cap \mathbb{Z}\right\}, \quad U=[-1 ; 1] .
$$

Proposition 2.1 (Ref. 11): Assume that $X_{N}$ is given either by (2.1) or $X_{N} \sim \mathrm{GREM}_{N}$. Fix some gauge invariant bivariate function $F_{N}: \Sigma_{N}^{2} \rightarrow \mathrm{R}$, and $q \in \mathbb{R}$.

Then, for all $\sigma^{\prime}, \tau^{\prime} \in \Sigma_{N}$, we have

$$
Z_{N}^{(p)}\left(\beta, q, \varepsilon, X_{N}, F_{N}\left(\cdot, \sigma^{\prime}\right)\right) \sim Z_{N}^{(p)}\left(\beta, q, \varepsilon, X_{N}, F_{N}\left(\cdot, \tau^{\prime}\right)\right) .
$$

In particular, the partial partition function (2.5) with $F_{N} \equiv R_{N}\left(\cdot, \sigma^{\prime}\right)$ has the same distribution as 
the partial partition function which corresponds to fixing the total magnetization (1.4), i.e.,

$$
Z_{N}^{(p)}\left(\beta, q, \varepsilon, X_{N}, R_{N}\left(\cdot, \sigma^{\prime}\right)\right) \sim Z_{N}^{(p)}(\beta, m, \varepsilon,) \equiv \sum_{\sigma:|m(\sigma)-q|<\varepsilon} \exp \left(\beta \sqrt{N} X_{N}(\sigma)\right) .
$$

Remark 2.1: The proposition obviously remains valid for the Hamiltonians $X_{N}$ given by the linear combinations of the $p$-spin Hamiltonians (2.1) with varying $p \in \mathbb{N}$.

Proof:

(1) If $X_{N}$ is defined by (2.1), then (2.7) follows due to the gauge invariance of (2.1) and $F_{N}$. Indeed, there exists $\mu \in \Sigma_{N}$ such that $\sigma^{\prime}=T_{\mu}\left(\tau^{\prime}\right)$. Define

$$
J_{i}^{(N, p, \mu)} \equiv J_{i}^{(N, p)} \mu_{i_{1}} \cdots \mu_{i_{p}} .
$$

Due to the symmetry of the joint distribution of $J^{(N, p)}$, we have

$$
\left\{X_{N}(\sigma)\right\}_{\sigma \in \Sigma_{N}} \sim\left\{\left.X_{N}(\sigma)\right|_{\left.J^{(N, p)}=J^{(N, p, \mu)}\right\}_{\sigma \in \Sigma_{N}}}\right.
$$

which implies (2.7).

(2) If $X_{N}=\mathrm{GREM}_{N}$, then, since $X_{N}$ is a Gaussian process, to prove the equality in distribution, it is enough to check that the covariance of $X_{N}$ is gauge symmetric. Equivalence (2.7) follows due to (1.2) and the fact that the lexicographic overlap (1.1) is gauge invariant.

The partial partition function (2.5) induces the restricted free energy in the usual way,

$$
p_{N}^{(p)}\left(\beta, q, \varepsilon, X_{N}, F_{N}\right) \equiv \frac{1}{N} \log Z_{N}^{(p)}\left(\beta, q, \varepsilon, X_{N}, F_{N}\right) .
$$

Given $\sigma^{(1)}, \sigma^{(2)} \in \Sigma_{N}$, let

$$
C_{N}\left(\sigma^{(1)}, \sigma^{(2)}\right) \equiv \mathbb{E}\left[X_{N}\left(\sigma^{(1)}\right) X_{N}\left(\sigma^{(2)}\right)\right], \quad \widetilde{C}_{N}\left(\sigma^{(1)}\right) \equiv C_{N}\left(\sigma^{(1)}, \sigma^{(1)}\right) .
$$

Define

$$
V_{N} \equiv\left\{C_{N}(\sigma, \sigma): \sigma \in \Sigma_{N}\right\}, \quad V \equiv \overline{\left(\bigcup_{N=1}^{\infty} V_{N}\right)} .
$$

The following result establishes a large deviation-type relation between the partial free energy and the full one.

Theorem 2.1: Assume $X_{N}=\left\{X_{N}(\sigma)\right\}_{\sigma \in \Sigma_{N}}$ is a centered Gaussian process and $F_{N}: \Sigma_{N} \rightarrow \mathbb{R}$ are such that, for all $N, M \in \mathbb{N}$, all $\sigma^{(1)}, \sigma^{(2)} \in \Sigma_{N}$, and all $\tau^{(1)}, \tau^{(2)} \in \Sigma_{M}$,

$$
\begin{aligned}
C_{N+M}\left(\sigma^{(1)}\left\|\tau^{(1)}, \sigma^{(2)}\right\| \tau^{(2)}\right) & \leq \frac{N}{N+M} C_{N}\left(\sigma^{(1)}, \sigma^{(2)}\right)+\frac{M}{N+M} C_{M}\left(\tau^{(1)}, \tau^{(2)}\right), \\
F_{N+M}\left(\sigma^{(1)} \| \tau^{(1)}\right) & \leq \frac{N}{N+M} F_{N}\left(\sigma^{(1)}\right)+\frac{M}{N+M} F_{M}\left(\tau^{(1)}\right) .
\end{aligned}
$$

Assume that $C_{N}$ and $F_{N}$ are bounded uniformly in $N$.

(1) The following holds:

$$
\begin{aligned}
p_{N}\left(\beta, X_{N}, F_{N}\right) & \equiv \frac{1}{N} \log \sum_{\sigma \in \Sigma_{N}} \exp \left(\beta \sqrt{N} X_{N}(\sigma)+N F_{N}(\sigma)\right) \\
& \underset{N \uparrow+\infty}{\longrightarrow} p(\beta, X, F), \quad \text { almost surely and in } L^{1} .
\end{aligned}
$$


(2) The limiting free energy $p(\beta, X, F)$ is almost surely deterministic.

(3) We have

$$
\begin{gathered}
\lim _{\varepsilon \downarrow+0} \lim _{N \uparrow+\infty} p_{N}^{(p)}\left(\beta, q, \varepsilon, X_{N}, F_{N}\right) \equiv p^{(p)}(\beta, q, X, F)=\sup _{v \in V} \inf _{\lambda \in \mathbb{R}, \gamma \in \mathbb{R}}(-\lambda q-\gamma v+p(\beta, X, \lambda F \\
+\gamma \widetilde{C})), \text { almost surely and in } L^{1} .
\end{gathered}
$$

(4) Finally,

$$
p(\beta, X, F)=\sup _{q \in U}\left(p^{(p)}(\beta, q, X, F)+q\right) .
$$

\section{Remark 2.2:}

(1) If there exists $\left\{\text { const }_{N} \in \mathbb{R}_{+}\right\}_{N=1}^{\infty}$ such that, for all $\sigma \in \Sigma_{N}$,

$$
\widetilde{C}_{N}(\sigma)=\text { const }_{N}
$$

then (2.12) simplifies to

$$
p^{(p)}(\beta, q, X, F)=\inf _{\lambda \in \mathbb{R}}(-\lambda q+p(\beta, X, \lambda F)), \text { almost surely and in } L^{1} .
$$

(2) Inequality (2.10) can alternatively be substituted by the assumption (see Guerra and Toninelli ${ }^{17}$ Theorem 1) that $F_{N}(\sigma)=f\left(S_{N}(\sigma)\right)$, where $f: \mathbb{R} \rightarrow \mathbb{R}, f \in C^{1}(\mathbb{R})$, and $S_{N}: \Sigma_{N} \rightarrow \mathbb{R}$ is the bounded function such that, for all $\sigma \in \Sigma_{N}, \tau \in \Sigma_{M}$,

$$
S_{N+M}(\sigma \| \tau)=\frac{N}{N+M} S_{N}(\sigma)+\frac{M}{N+M} S_{M}(\tau)
$$

(3) It is easy to check that the assumptions of Proposition 2.1 are fulfilled, e.g., for

$$
X_{N} \equiv c_{1} \mathrm{GREM}_{N}+c_{2} \mathrm{SK}_{N}^{(p)},
$$

and

$$
F_{N}(\cdot) \equiv f_{1}\left(R_{N}\left(\cdot, \sigma^{(N)}\right)\right)+f_{2}\left(q_{\mathrm{L}}\left(\cdot, \sigma^{(N)}\right)\right),
$$

where $\sigma^{(N)} \in \Sigma_{N}, c_{1}, c_{2} \in \mathbb{R}$, and $f_{1}, f_{2}: \mathbb{R} \rightarrow \mathbb{R}$, such that $f_{1} \in C^{1}(\mathbb{R}), f_{2}$ is convex. Note that in this case, due to Proposition 2.1, the free energies (2.11) and (2.12) do not depend on the choice of the sequence $\left\{\sigma^{(N)}\right\}_{N=1}^{\infty} \subset \Sigma_{N}$.

Proof: Similarly to Contucci et al. ${ }^{18}$ Theorem 1 and Guerra and Toninelli ${ }^{17}$ Theorem 1 , we obtain (2.11). Then (2.11) implies that

$$
p\left(\beta, X_{N}, \lambda F_{N}+\gamma \widetilde{C}_{N}\right) \underset{N \uparrow+\infty}{\longrightarrow} p(\beta, X, \lambda F+\gamma \widetilde{C}), \quad \text { almost surely and in } L^{1}
$$

Hence, we can apply the quenched large deviation results Bovier and Klimovsky ${ }^{19}$ Theorems 3.1 and 3.2 which readily yield (2.13) and (2.12) [or (2.15)], in the case of (2.14).

Remark 2.3: Derrida and Gardner ${ }^{11}$ sketched a calculation of the free energy defined in (2.11) in the following case:

$$
F_{N}=q_{L} \text { and } X_{N}=\mathrm{GREM}_{N} .
$$

This case is easier than the case (1.6) we are considering here, since both $q_{L}$ and $\mathrm{GREM}_{N}$ have lexicographic nature, $c f$. (1.2) and (1.1). 


\section{THE REM WITH EXTERNAL FIELD REVISITED}

In this section, we recall some known results on the limiting free energy of the REM with external field. However, we give some new proofs of these results which illustrate the approach of Sec. II. Moreover, we prove the weak limit theorem for the ground state and for the partition function of the REM with external field.

Recall that the REM corresponds to the case $n=1$ in (1.12). This implies that the process $X$ is simply a family of $2^{N}$ independently and identically distributed standard Gaussian random variables. To emphasize this situation we shall write $\operatorname{REM}(\sigma)$ instead of $\operatorname{GREM}(\sigma)$.

\section{A. Free energy and ground state}

Let us start by recalling the following well-known result on the REM.

Theorem 3.1 (Derrida, ${ }^{20}$ Eisele, $^{21}$ and Olivieri and Picco ${ }^{22}$ ): Assume that $n=1$ and let $p(\beta, h)$ be given by (1.6). The following assertions hold.

(1) We have

$$
\lim _{N \rightarrow \infty} p_{N}(\beta, 0)=\left\{\begin{array}{ll}
\frac{\beta^{2}}{2}+\log 2, & \beta \leq \sqrt{2 \log 2}, \\
\beta \sqrt{2 \log 2}, & \beta \geq \sqrt{2 \log 2},
\end{array} \quad \text { almost surely and in } L^{1} .\right.
$$

(2) For all $\beta \geq \sqrt{2 \log 2}$ and $N \in \mathbb{N}$, we have

$$
0 \leq \mathbb{E}\left[p_{N}(\beta, 0)\right] \leq \beta \sqrt{2 \log 2} .
$$

See, e.g., Bovier ${ }^{6}$ Theorem 9.1.2 for a short proof.

Given $k \in[0 ; N] \cap \mathbb{N}$, define the set of configurations having a given magnetization

$$
\Sigma_{N, k} \equiv\left\{\sigma \in \Sigma_{N}: \sum_{i=1}^{N} \sigma_{i}=N-2 k\right\}
$$

Lemma 3.1: Set $t_{k, N} \equiv N-2 k / N$. Given any $\varepsilon>0$, uniformly in $k \in[0 ; N] \cap \mathbb{N}$ such that

$$
t_{k, N} \in[-1+\varepsilon ; 1-\varepsilon]
$$

we have the following asymptotics:

$$
\left(\begin{array}{l}
N \\
k
\end{array}\right) \underset{N \uparrow+\infty}{=} \sqrt{\frac{2}{\pi}} \frac{2^{N} e^{-N I\left(t_{k, N}\right)}}{\sqrt{N\left(1-t_{k, N}^{2}\right)}}\left(1+\frac{1}{N}\left(\frac{1}{12}+\frac{1}{3\left(1-t_{k, N}^{2}\right)}\right)+\mathcal{O}\left(\frac{1}{N^{2}}\right)\right) .
$$

Proof: A standard exercise on Stirling's formula.

Theorem 3.2 (Dorlas and Wedagedera ${ }^{23}$ ): Assume that $n=1$ (the REM case) and let $p(\beta, h)$ be given by (1.6). We have

$$
\begin{aligned}
p(\beta, h) & \equiv \lim _{N \rightarrow \infty} p_{N}(\beta, h) \\
& =\left\{\begin{array}{l}
\log 2+\log \operatorname{ch} \beta h+\frac{\beta^{2}}{2}, \quad \beta \leq \sqrt{2\left(\log 2-I\left(t_{*}\right)\right)} \equiv \beta_{0} \\
\beta\left(\sqrt{2\left(\log 2-I\left(t_{*}\right)\right)}+h t_{*}\right), \quad \beta \geq \sqrt{2\left(\log 2-I\left(t_{*}\right)\right)}
\end{array}, \quad \text { almost surely and in } L^{1},\right.
\end{aligned}
$$

and $t_{*} \in(-1 ; 1)$ is a unique maximizer of the following concave function:

$$
(-1 ; 1) \ni t \mapsto h t+\sqrt{2(\log 2-I(t))} .
$$


Proof: For the sake of completeness, we give a short proof based on (the ideas of) Theorem 2.1. Put

$$
M_{k, N} \equiv \begin{cases}\left\lfloor\log _{2}\left(\begin{array}{l}
N \\
k
\end{array}\right)\right\rfloor, & k \in[1 ; N-1] \cap \mathbb{N}, \\
1, & k \in\{0, N\}\end{cases}
$$

where $\lfloor x\rfloor$ denotes the largest integer smaller than $x$. Consider the free energy [cf. (2.8)] of the REM of volume $M_{k, N}$,

$$
p_{k, N}(\beta) \equiv \frac{1}{M_{k, N}} \log \sum_{\sigma \in \Sigma_{N}^{k}} \exp \left(\beta M_{k, N}^{1 / 2} \operatorname{REM}(\sigma)\right),
$$

where $\operatorname{REM} \equiv\{\operatorname{REM}(\sigma)\}_{\sigma \in \Sigma_{N}}$ is the family of standard independently and identically distributed Gaussian random variables. Let

$$
\tilde{p}_{k, N}(\beta) \equiv \frac{M_{k, N}}{N} p_{k, N}\left(\left(\frac{N}{M_{k, N}}\right)^{1 / 2} \beta\right) .
$$

Note that (3.6) is the restricted free energy [cf. (2.8)] of the REM, where the restriction is imposed by the total magnetization (1.4) given by $t_{k, N}$.

We claim that the family of functions $\mathcal{P} \equiv\left\{\mathbb{E}\left[p_{N}(\cdot)\right]\right\}_{N \in \mathbb{N}}$ is uniformly Lipschitzian. Indeed, uniformly in $\beta \geq 0$, we have

$$
\partial_{\beta} \mathbb{E}\left[p_{N}(\beta)\right]=N^{-1 / 2} \mathbb{E}\left[\mathcal{G}_{N}(\beta, 0)\left[X_{N}(\sigma)\right]\right] \leq N^{-1 / 2} \mathbb{E}\left[\max _{\sigma \in \Sigma_{N}} X(\sigma)\right]_{N \uparrow+\infty}^{\rightarrow} \sqrt{2 \log 2} .
$$

Hence, the family $\mathcal{P}$ has uniformly bounded first derivatives.

Given $t \in(-1 ; 1)$ and $t_{k_{N}, N} \in U_{N}$ [cf. (2.6)] such that $\lim _{N \uparrow+\infty} t_{k_{N}, N}=t$, using (3.4), we have

$$
\lim _{N \uparrow+\infty} \frac{M_{k_{N}, N}}{N}=1-I(t) \log _{2} e .
$$

Using (3.7) and the uniform Lipschitzianity of the family $\mathcal{P}$, we get

$$
\lim _{N \uparrow+\infty} \widetilde{p}_{k_{N}, N}(\beta)=\left(1-I(t) \log _{2} e\right) p\left(\frac{\beta}{\sqrt{1-I(t) \log _{2} e}}\right) .
$$

Combining (3.8) with (2.13) and (2.15), we get

$$
p(\beta, h)=\max _{t \in[-1 ; 1]}\left\{t \beta h+\left(1-I(t) \log _{2} e\right) p\left(\frac{\beta}{\sqrt{1-I(t) \log _{2} e}}\right)\right\} .
$$

To find the maximum in (3.9), we consider two cases.

(1) If $\beta \leq \sqrt{2\left(\log 2-I\left(t_{*}\right)\right)}$, then according to (3.1), we have

$$
p\left(\frac{\beta}{\sqrt{1-I(t) \log _{2} e}}\right)=\log 2+\frac{\beta^{2}}{2\left(1-I(t) \log _{2} e\right)} .
$$

Hence, (3.9) implies

$$
p(\beta, h)=\max _{t \in[-1 ; 1]}\left\{t \beta h+\frac{\beta^{2}}{2}+\log 2-I(t)\right\}=\log 2+\log \operatorname{ch} \beta h+\frac{\beta^{2}}{2},
$$

where the last equality is due to the fact that the expression in the curly brackets is concave and, hence, the maximum is attained at a stationary point. The stationarity condition reads 


$$
t=t_{0}(\beta, h) \equiv \tanh \beta h .
$$

It is easy to check that the following identity holds:

$$
I(t)=t \tanh ^{-1} t-\log \operatorname{ch~tanh}^{-1} t .
$$

Combining (3.12) and (3.11), we get (3.10).

(2) If $\beta \geq \sqrt{2\left(\log 2-I\left(t_{*}\right)\right)}$, then again by (3.1), we have

$$
p\left(\frac{\beta}{\sqrt{1-I(t) \log _{2} e}}\right)=\frac{\beta \sqrt{2 \log 2}}{\sqrt{1-I(t) \log _{2} e}} .
$$

Hence, (3.9) transforms to

$$
p(\beta, h)=\max _{t \in[-1 ; 1]}\{t \beta h+\beta \sqrt{2(\log 2-I(t))}\}=\beta\left(\sqrt{2\left(\log 2-I\left(t_{*}\right)\right)}+h t_{*}\right),
$$

where the last equality is due to the concavity of the expression in the curly brackets. Combining (3.10) and (3.13), we get (3.5).

Remark 3.1: We note that due to the continuity of the free energy as a function of $\beta$, we have at the freezing temperature $\beta_{0}$,

$$
t_{0}\left(\beta_{0}, h\right)=t_{*}(h) .
$$

Theorem 3.2 suggests that the following holds.

Theorem 3.3: Under the assumptions of Theorem 3.2, we have

$$
\lim _{N \uparrow+\infty} \frac{1}{\sqrt{N}} \max _{\sigma \in \Sigma_{N}} X_{N}(h, \sigma)=\sqrt{2\left(\log 2-I\left(t_{*}\right)\right)}+h t_{*}, \quad \text { almost surely and in } L^{1} .
$$

Proof: We have

$$
\frac{1}{\beta} p_{N}(\beta) \leq \frac{1}{N} \log \left(N \beta \sqrt{N} \max _{\sigma \in \Sigma_{N}} X_{N}(h, \sigma)\right)=\frac{\log N}{\beta N}+\frac{1}{\sqrt{N}} \max _{\sigma \in \Sigma_{N}} X_{N}(h, \sigma) .
$$

In view of (3.5), relation (3.16) readily implies that

$$
\sqrt{2\left(\log 2-I\left(t_{*}\right)\right)}+h t_{*} \leq \underline{\lim _{N \uparrow+\infty}} N^{-1 / 2} \max _{\sigma \in \Sigma_{N}} X_{N}(h, \sigma) .
$$

We also have

$$
\frac{1}{\beta} p_{N}(\beta) \geq \frac{1}{\sqrt{N}} \max _{\sigma \in \Sigma_{N}} X_{N}(h, \sigma),
$$

which combined again with (3.5) implies that

$$
\sqrt{2\left(\log 2-I\left(t_{*}\right)\right)}+h t_{*} \geq \varlimsup_{N \uparrow+\infty} N^{-1 / 2} \max _{\sigma \in \Sigma_{N}} X_{N}(h, \sigma) .
$$

Due to the standard concentration of Gaussian measure [e.g., Ledoux $\left.{ }^{24}(2.35)\right]$ and the fact that the free energy (1.6) is Lipschitzian with the constant $\beta \sqrt{N}$ as a function of $X_{N}(h, \cdot)$ with respect to the Euclidean topology, the bounds (3.17) and (3.18) combined with the Borell-Cantelli lemma give the convergence (3.15).

\section{B. Fluctuations of the ground state}

In this subsection, we shall study the limiting distribution of the point process generated by the properly rescaled process of the energy levels, i.e., (1.25). 
Proof of Theorem 1.1: Let us denote

$$
\mathcal{E}_{N}(h) \equiv \sum_{\sigma \in \Sigma_{N}} \delta_{u_{N, h}^{-1}\left(X_{N}(h, \sigma)\right)}
$$

We treat $\mathcal{E}_{N}(h)$ as a random pure point measure on $\mathbb{R}$. Given some test function $\varphi \in C_{0}^{+}(\mathbb{R})$ (i.e., a non-negative function with compact support), consider the Laplace transform of (3.19) corresponding to $\varphi$,

$$
\begin{aligned}
L_{\mathcal{E}_{N}(h)}(\varphi) & \equiv \mathbb{E}\left[\exp \left\{-\sum_{\sigma \in \Sigma_{N}} \varphi\left(u_{N, h}^{-1}\left(X_{N}(h, \sigma)\right)\right)\right\}\right. \\
& =\prod_{k=0}^{N}\left\{\frac{1}{2 \pi} \int_{\mathbb{R}} \exp \left(-\varphi\left(u_{N, h}^{-1}\left(x+\frac{h}{\sqrt{N}}(N-2 k)\right)\right)-\frac{x^{2}}{2}\right) d x\right\}^{\left(\begin{array}{c}
N \\
k
\end{array}\right)} .
\end{aligned}
$$

Introduce the new integration variables $y=u_{N, h}^{-1}(x+h / \sqrt{N}(N-2 k))$. We have

$$
\begin{aligned}
(3.20) & =\prod_{k=0}^{N}\left\{\frac{A_{N}(h)}{2 \pi} \int_{\mathbb{R}} \exp \left(-\varphi(y)-\frac{1}{2}\left(u_{N, h}(y)-\frac{h}{\sqrt{N}}(N-2 k)\right)^{2}\right) d y\right\}^{\left(\begin{array}{l}
N \\
k
\end{array}\right)} \\
& =\exp \left\{\sum_{k=0}^{N}\left(\begin{array}{l}
N \\
k
\end{array}\right) \log \left(1-\frac{A_{N}(h)}{\sqrt{2 \pi}} \int_{\mathbb{R}}\left(1-e^{-\varphi(y)}\right) \exp \left[-\frac{1}{2}\left(u_{N, h}(y)-\frac{h}{\sqrt{N}}(N-2 k)\right)^{2}\right]\right) .\right.
\end{aligned}
$$

Note that the integration in (3.21) is actually performed over $y \in \operatorname{supp} \varphi$, since the integrand is zero on the complement of the support. It is easy to check that uniformly in $y \in \operatorname{supp} \varphi$ the integrand in (3.21) and, hence, the integral itself is exponentially small (as $N \uparrow+\infty$ ). Consequently, we have

$$
\begin{aligned}
(3.21) \underset{N \uparrow+\infty}{=} & \exp \left\{-\int_{\operatorname{supp} \varphi}\left(1-e^{-\varphi(y)}\right) \sum_{k=0}^{N}\left(\begin{array}{l}
N \\
k
\end{array}\right) \frac{A_{N}(h)}{\sqrt{2 \pi}} \exp \left[-\frac{1}{2}\left(u_{N, h}(y)-\frac{h}{\sqrt{N}}(N-2 k)\right)^{2}\right]\right) \\
& \times(1+o(1))\} .
\end{aligned}
$$

Denote $t_{k, N} \equiv N-2 k / N$. Using Lemma 3.1, we get

$$
\begin{aligned}
(3.22) \underset{N \uparrow+\infty}{=} & \exp \left\{-(1+o(1)) \int_{\operatorname{supp} \varphi}\left(1-e^{-\varphi(y)}\right) \sum_{k=0}^{N} \frac{A_{N}(h)}{\pi\left(N\left(1-t_{k, N}^{2}\right)\right)^{1 / 2}} \exp \left[N\left(\log 2-I\left(t_{k, N}\right)\right)\right.\right. \\
& \left.\left.\left.-\frac{1}{2}\left(u_{N, h}(y)-h t_{k, N} \sqrt{N}\right)^{2}\right]\right)\right\} .
\end{aligned}
$$

Note that despite the fact that Lemma 3.1 is valid only for $t_{k, N} \in[-1+\varepsilon ; 1-\varepsilon]$, we can still write (3.23), since the both following sums are negligible:

$$
\begin{aligned}
0 & \leq \sum_{k: t_{k, N} \in([-1 ;-1+\varepsilon] \cup[1-\varepsilon, 1])} \frac{A_{N}(h)}{\pi\left(N\left(1-t_{k, N}^{2}\right)\right)^{1 / 2}} \exp \left[N\left(\log 2-I\left(t_{k, N}\right)\right)-\frac{1}{2}\left(u_{N, h}(y)-h t_{k, N} \sqrt{N}\right)^{2}\right] \\
& \leq K N \exp (-L N)
\end{aligned}
$$

and 


$$
0 \leq \sum_{k: t_{k, N} \in([-1 ;-1+\varepsilon] \cup[1-\varepsilon, 1])}\left(\begin{array}{l}
N \\
k
\end{array}\right) \frac{A_{N}(h)}{\sqrt{2 \pi}} \exp \left[-\frac{1}{2}\left(u_{N, h}(y)-h t_{k, N} \sqrt{N}\right)^{2}\right] \leq K N \exp (-L N) .
$$

Consider the sum appearing in (3.23),

$$
S_{N}(h, y) \equiv \sum_{k=0}^{N} \frac{A_{N}(h)}{\pi\left(N\left(1-t_{k, N}^{2}\right)\right)^{1 / 2}} \exp \left[N\left(\log 2-I\left(t_{k, N}\right)\right)-\frac{1}{2}\left(u_{N, h}(y)-h t_{k, N} \sqrt{N}\right)^{2}\right] .
$$

Introduce the functions $f_{N}, g_{N}:[-1 ; 1] \rightarrow \mathrm{R}$ as

$$
\begin{gathered}
f_{N}(t) \equiv I(t)+\frac{1}{2}\left(\frac{u_{N, h}(y)}{\sqrt{N}}-h t\right)^{2}-\log 2, \\
g_{N}(t) \equiv \frac{A_{N}(h)}{\pi\left(N\left(1-t^{2}\right)\right)^{1 / 2}} .
\end{gathered}
$$

Note that definition (1.14) implies

$$
I^{\prime}\left(t_{*}\right)=h \mu\left(t_{*}\right) .
$$

A straightforward computation using (1.15), (1.16), and (3.25) gives

$$
\begin{gathered}
f_{N}^{\prime \prime}(t)=I^{\prime \prime}(t)+h>0, \\
f_{N}^{\prime}\left(t_{*}\right)=-\frac{h}{\left(2 \mu\left(t_{*}\right) N\right)}\left[2 y+\log \left(\frac{I^{\prime \prime}\left(t_{*}\right)+h}{4 \pi\left(1-t_{*}^{2}\right)\left(\log 2-I\left(t_{*}\right)\right) N}\right)\right]=O\left(\frac{\log N}{N}\right), \\
f_{N}\left(t_{*}\right)=-\frac{1}{N}\left[y+\frac{1}{2} \log \left(\frac{I^{\prime \prime}\left(t_{*}\right)+h}{4 \pi\left(1-t_{*}^{2}\right)\left(\log 2-I\left(t_{*}\right)\right) N}\right)\right]+o\left(\frac{1}{N}\right) .
\end{gathered}
$$

Hence, since (3.27) vanishes even after being multiplied by $\sqrt{N}$, (3.27) is negligible for the purposes of the asymptotic Laplace principle. This readily implies that uniformly in $y \in \operatorname{supp} \varphi$,

$$
S_{N}(h, y) \underset{N \uparrow+\infty}{\sim} \frac{N g_{N}\left(t_{*}\right)}{2}\left(\frac{2 \pi f_{N}^{\prime \prime}\left(t_{*}\right)}{N}\right)^{1 / 2} \exp \left[N f_{N}\left(t_{*}\right)\right]
$$

Using (3.26)-(3.28) in the right hand side of (3.29), we obtain that uniformly in $y \in \operatorname{supp} \varphi$,

$$
S_{N}(h, y) \underset{N \uparrow+\infty}{\sim} \exp (-y) .
$$

Finally, combining (3.30) and (3.23), we obtain

$$
\lim _{N \uparrow+\infty} L_{\mathcal{E}_{N}(h)}(\varphi)=\exp \left\{-\int_{\mathbb{R}}\left(1-e^{-\varphi(y)}\right) e^{-y} d y\right\} .
$$

The right hand side of (3.31) is the Laplace transform of $\operatorname{PPP}\left(e^{-x} d x, x \in \mathbb{R}\right)$. Then a standard result implies the claim (1.19).

\section{Fluctuations of the partition function}

In this subsection, we compute the weak limiting distribution of the partition function under the natural scaling induced by (1.17). Define 


$$
\begin{gathered}
C_{N}(\beta, h) \equiv \exp \left[\beta M(h) N+\frac{\beta}{2 \mu\left(t_{*}\right)} \log \left(\frac{I^{\prime \prime}\left(t_{*}\right)+h}{4 \pi\left(1-t_{*}^{2}\right)\left(\log 2-I\left(t_{*}\right)\right) N}\right)\right], \\
D_{N}(\beta, h) \equiv \operatorname{ch}^{-2 / 3}(\beta h) \exp \left[N\left(\log 2+\log \operatorname{ch} \beta h+\frac{\beta^{2}}{2}\right)\right], \\
\alpha(\beta, h) \equiv \frac{\beta}{\mu\left(t_{*}\right)} .
\end{gathered}
$$

Theorem 3.4: If $\beta>\mu\left(t_{*}\right)$, then

$$
\frac{Z_{N}(\beta, h)}{C_{N}(\beta, h)} \underset{N \uparrow+\infty}{\stackrel{w}{\longrightarrow}} \int_{\mathbb{R}} e^{\alpha(\beta, h) x} d \mathcal{P}^{(1)}(x)
$$

If $\beta<\mu\left(t_{*}\right)$, then

$$
\frac{Z_{N}(\beta, h)}{D_{N}(\beta, h)} \underset{N \uparrow+\infty}{\stackrel{w}{\longrightarrow}} 1 .
$$

Proof: This is a specialization of Theorem 1.3 which is proven in Sec. IV B.

\section{THE GREM WITH EXTERNAL FIELD}

In this section, we obtain the main results of the paper concerning the GREM with external field. We prove the limit theorems for the distribution of the partition function and that of the ground state. As a simple consequence of these fluctuation results, we obtain an explicit formula for the free energy of the GREM with external field.

\section{A. Fluctuations of the ground state}

As in the REM, we start from the ground state fluctuations (cf. Theorem 1.1). The following is the main technical result of this section that shows exactly in which situations the GREM with external field has the same scaling limit behavior as the REM with external field.

Proposition 4.1: Either of the following two cases holds.

(1) If, for all $l \in[2 ; n] \cap \mathbb{N}$,

$$
\frac{\log 2-I\left(t_{*}\left(\theta_{l, n}^{-1 / 2} h\right)\right)}{\log 2-I\left(t_{*}(h)\right)}<\theta_{l, n}
$$

then we have

$$
\sum_{\sigma \in \Sigma_{N}} \delta_{u_{N, h}^{-1}\left(X_{N}(h, \sigma)\right)}^{\stackrel{w}{\longrightarrow}} \operatorname{PPP}\left(e^{-x}, x \in \mathbb{R}^{d}\right) .
$$

(2) If, for all $l \in[2, \ldots, n] \cap \mathbb{N}$,

$$
\frac{\log 2-I\left(t_{*}\left(\theta_{l, n}^{-1 / 2} h\right)\right)}{\log 2-I\left(t_{*}(h)\right)} \leq \theta_{l, n}
$$

and there exists (at least one) $l_{0} \in[2 ; n] \cap \mathbb{N}$,

$$
\frac{\log 2-I\left(t_{*}\left(\theta_{l_{0}, n}^{-1 / 2} h\right)\right)}{\log 2-I\left(t_{*}(h)\right)}=\theta_{l_{0}, n},
$$

then there exists the constant $K=K(\rho, h) \in(0 ; 1)$, such that 


$$
\sum_{\sigma \in \Sigma_{N}} \delta_{u_{N, h}^{-1}\left(X_{N}(h, \sigma)\right)}^{\stackrel{w}{\longrightarrow}} \underset{N \uparrow+\infty}{\longrightarrow} \operatorname{PPP}\left(K e^{-x}, x \in \mathbb{R}\right) .
$$

Remark 4.1: If condition (4.3) is violated, i.e., there exists $l_{0} \in[2 ; n] \cap \mathbb{N}$ such that

$$
\frac{\log 2-I\left(t_{*}\left(\theta_{l, n}^{-1 / 2} h\right)\right)}{\log 2-I\left(t_{*}(h)\right)}>\theta_{l, n}
$$

then the REM scaling [cf. (1.19) and (1.17)] is too strong to reveal the structure of the ground state fluctuations of the GREM. Theorem 1.2 shows how the scaling and the limiting object should be modified to capture the fluctuations of the GREM in this regime.

Proof:

(1) Denote $N_{l} \equiv \Delta x_{l} N$, for $l \in[1 ; n]$. We fix arbitrary test function $\varphi \in C_{\mathrm{K}}^{+}(\mathbb{R})$, i.e., a nonnegative function with compact support. Consider the Laplace transform $L_{\mathcal{E}_{N}(h)}(\varphi)$ of the random measure $\mathcal{E}_{N}(h)$ evaluated on the test function $\varphi$ :

$$
L_{\mathcal{E}_{N}(h)}(\varphi) \equiv \mathbb{E}\left[\exp \left(-\sum_{\sigma \in \Sigma_{N}}\left(\varphi \circ u_{N, h}^{-1}\right)\left(X_{N}(h, \sigma)\right)\right)\right]=\mathbb{E}\left[\prod_{\sigma \in \Sigma_{N}} \exp \left(-\left(\varphi \circ u_{N, h}^{-1}\right)\left(X_{N}(h, \sigma)\right)\right)\right] .
$$

Consider also the family of independently and identically distributed standard Gaussian random variables,

$$
\left\{X\left(\sigma^{(l)}, \sigma^{(2)}, \ldots, \sigma^{(n)}\right) \mid l \in[1 ; n] \cap \mathbb{N}, \sigma^{(l)} \in \Sigma_{N_{l}}, \ldots, \sigma^{(n)} \in \Sigma_{N_{n}}\right\} .
$$

Given $l \in[1 ; n] \cap \mathbb{N}$ and $y \in \mathbb{R}$, define

$$
\begin{aligned}
& L_{N}(l, v) \equiv \mathbb{E}\left[\prod _ { \sigma ^ { ( l ) } \| \ldots \| \sigma ^ { ( n ) } \in \Sigma _ { ( 1 - x _ { l - 1 } ) N } } \operatorname { e x p } \left(-\varphi \circ u_{N, h}^{-1}\left(v+a_{l} X\left(\sigma^{(l)}\right)+\cdots+a_{n} X\left(\sigma^{(l)}, \ldots, \sigma^{(n)}\right)\right.\right.\right. \\
&\left.\left.\left.+h\left(1-x_{l-1}\right) \sqrt{N} m\left(\sigma^{(l)}, \ldots, \sigma^{(n)}\right)\right)\right)\right] .
\end{aligned}
$$

We readily have

$$
L_{\mathcal{E}_{N}(h)}(\varphi)=L_{N}(1,0) .
$$

Due to the treelike structure of the GREM, for $l \in[1 ; n-1] \cap \mathbb{N}$, we have the following recursion:

$$
L_{N}(l, v)=\prod_{\sigma^{(l)} \in \Sigma_{N_{l}}} \mathbb{E}\left[L_{N}\left(l+1, v+a_{l} X+h \Delta x_{l} \sqrt{N} m\left(\sigma^{(l)}\right)\right)\right],
$$

where $X$ is a standard Gaussian random variable. Introduce the following quantities:

$$
Y_{N}(h, y, v, t, l) \equiv u_{N, h}(y)-h\left(1-x_{l-1}\right) \sqrt{N} t-v .
$$

We claim that, for any $l \in[1 ; n] \cap \mathbb{N}$, uniformly in $v \in \mathbb{R}$ satisfying

$$
v \leq \sqrt{N}\left(M(h)-\delta-\left(1-q_{l-1}\right) \mu(h)-h\left(1-x_{l-1}\right) t_{*}\left(\theta_{l, n}^{-1 / 2} h\right)\right),
$$

we have 


$$
\begin{aligned}
\log L_{N}(l, v) \underset{N \uparrow+\infty}{\sim} & -\frac{A_{N}(h)}{\sqrt{2 \pi\left(1-q_{l-1}\right)}} \sum_{k=0}^{\left(1-x_{l-1}\right) N}\left(\left(\begin{array}{c}
\left(1-x_{l-1}\right) N \\
k
\end{array}\right)\right. \\
& \left.\times \int_{\mathbb{R}}\left(1-e^{-\varphi(y)}\right) \exp \left[-\frac{1}{2\left(1-q_{l-1}\right)} Y_{N}\left(h, y, v, t_{k,\left(1-x_{l-1}\right) N}, l\right)^{2}\right] d y\right) .
\end{aligned}
$$

We shall prove (4.11) by a decreasing induction in $l$ starting from $l=n$.

(2) The base of induction is a minor modification of the proof of Theorem 1.1. By the definition (4.8) and independence, we have

$$
L_{N}(n, v)=\prod_{k=0}^{N_{n}}\left(\mathbb{E} \exp \left[-\left(\varphi \circ u_{h, N}^{-1}\right)\left(a_{n} X+h \Delta x_{n} \sqrt{N} t_{k, N_{n}}+v\right)\right]\right)\left(\begin{array}{c}
N_{n} \\
k
\end{array}\right) .
$$

For fixed $k \in\left[0 ; N_{n}\right] \cap \mathbb{Z}$,

$$
\begin{aligned}
\mathbb{E}\left[\exp \left(-\left(\varphi \circ u_{N, h}^{-1}\right)\left(a_{n} X+h \Delta x_{n} \sqrt{N} t_{k, N}+v\right)\right)\right] \\
=(2 \pi)^{-1 / 2} \int_{\mathbb{R}} d x \exp \left[-x^{2} / 2-\left(\varphi \circ u_{N, h}^{-1}\right)\left(a_{n} x+h \Delta x_{n} \sqrt{N} t_{k, N}+v\right)\right] .
\end{aligned}
$$

We introduce in (4.13) the new integration variable,

$$
y \equiv u_{N, h}^{-1}\left(a_{n} x+h \Delta x_{n} \sqrt{N} t_{k, N_{n}}+v\right)
$$

Using the change of variables (4.14), we get that the right hand side of (4.13) is equal to

$$
\frac{A_{N}(h)}{\sqrt{2 \pi} a_{n}} \int_{\mathrm{R}} d y \exp \left[-\frac{1}{2 a_{n}^{2}} Y_{N}\left(h, y, v, t_{k, N_{n}}, n\right)^{2}-\varphi(y)\right] .
$$

Combining (4.12) and (4.15), we get

$$
\begin{aligned}
L_{N}(n, v) & =\prod_{k=0}^{N_{n}}\left(\frac{A_{N}(h)}{\sqrt{2 \pi} a_{n}} \int_{\mathbb{R}} d y \exp \left[-\frac{1}{2 a_{n}^{2}} Y_{N}\left(h, y, v, t_{k, N_{n}}, n\right)^{2}-\varphi(y)\right]\right)\left(\begin{array}{c}
N_{n} \\
k
\end{array}\right) \\
& =\prod_{k=0}^{N_{n}}\left(1-\frac{A_{N}(h)}{\sqrt{2 \pi} a_{n}} \int_{\mathbb{R}} d y\left(1-e^{-\varphi(y)}\right) \exp \left[-\frac{1}{2 a_{n}^{2}} Y_{N}\left(h, y, v, t_{k, N_{n}}, n\right)^{2}\right]\right)^{\left(\begin{array}{c}
N_{n} \\
k
\end{array}\right)} .
\end{aligned}
$$

Define

$$
V_{N}(h, v, t, n) \equiv \frac{A_{N}(h)}{\sqrt{2 \pi} a_{n}} \int_{\mathrm{R}} d y\left(1-e^{-\varphi(y)}\right) \exp \left[-\frac{1}{2 a_{n}^{2}} Y_{N}(h, y, v, t, n)^{2}\right] .
$$

Given any small enough $\delta>0$, it straightforward to show that uniformly in $v \in \mathbb{R}$ such that

$$
v \leq \sqrt{N}\left(M(h)-h \Delta x_{n} t_{*}\left(h \theta_{n-1, n}^{-1 / 2}\right)-\delta\right),
$$

we have

$$
L_{N}(n, v)=\prod_{N \uparrow+\infty}^{N_{n}}\left(1-\left(\begin{array}{c}
N_{n} \\
k
\end{array}\right) V_{N}\left(h, v, t_{k, N_{n}}, n\right)\right)\left(1+\mathcal{O}\left(e^{-C N}\right)\right) .
$$

Indeed, we have 


$$
\exp \left[-\frac{1}{2 a_{n}^{2}} Y_{N}\left(h, y, v, t_{k, N_{n}}, n\right)^{2}\right] \leq \exp \left[-N_{n}\left(\log 2-I\left(t_{n}\right)\right)\right]
$$

Next, using the fact that $\left(1-e^{-\varphi(\cdot)}\right) \in C_{K}^{+}(\mathbb{R})$, we get for some $C>0$,

$$
\int_{\mathrm{R}} d y\left(1-e^{-\varphi(y)}\right) \exp \left[-\frac{1}{2 a_{n}^{2}} Y_{N}\left(h, y, v, t_{k, N_{n}}, n\right)^{2}\right] \leq C \exp \left[-N_{n}\left(\log 2-I\left(t_{n}\right)\right)\right]
$$

Applying the elementary bounds

$$
x-x^{2} \leq \log (1+x) \leq x \quad \text { for }|x|<\frac{1}{2}
$$

to

$$
x \equiv-\frac{A_{N}(h)}{\sqrt{2 \pi} a_{n}} \int_{\mathrm{R}} d y\left(1-e^{-\varphi(y)}\right) \exp \left[-\frac{1}{2 a_{n}^{2}} Y_{N}\left(h, y, v, t_{k, N_{n}}, n\right)^{2}\right],
$$

and using the fact that, due to (3.4), there exists $C>0$ such that uniformly in $k \in\left[1 ; N_{n}\right] \cap \mathbb{N}$,

$$
x^{2} \leq \exp \left[-2 N_{n}\left(\log 2-I\left(t_{n}\right)\right)\right]\left(\begin{array}{c}
N_{n} \\
k
\end{array}\right) \leq e^{-C N},
$$

we get (4.16) and, consequently, (4.11) holds for $l=n$.

(3) For simplicity of presentation, we prove only the induction step $l=n \rightsquigarrow l=n-1$. Due to (4.10), we have

$$
L_{N}(n-1, v)=\prod_{k_{n-1}=0}^{N_{n-1}} \mathbb{E}\left[L_{N}\left(n, v+a_{n-1} X+h \Delta x_{n-1} \sqrt{N} t_{k_{n-1}, N_{n-1}}\right)\right]\left(\begin{array}{c}
N_{n-1} \\
k_{n-1}
\end{array}\right) .
$$

Define

$$
t\left(k_{n}, k_{n-1}\right) \equiv \frac{1}{1-x_{l-2}}\left(\Delta x_{n} t_{k_{n}, N_{n}}+\Delta x_{n-1} t_{k_{n-1}, N_{n-1}}\right) .
$$

Fix an arbitrary $\delta>0$ and $\varepsilon>0$. Due to (4.11) with $l=n$, there exists some $C>0$, such that uniformly for all $k_{n}, k_{n-1}$ with

$$
t_{k_{n}, k_{n-1}} \in\left\{t \in[-1 ; 1]:\left|t_{*}\left(\theta_{n-1, n}^{-1 / 2} h\right)-t_{k_{n}, k_{n-1}}\right| \leq \varepsilon\right\},
$$

and uniformly for all $v, x \in \mathbb{R}$ satisfying

$$
\Delta x_{n}\left(\log 2-I\left(t_{k_{n}, N_{n}}\right)\right) \leq \frac{1}{2 a_{n}^{2}}\left(M(h)-\delta-a_{n-1} x-N^{-1 / 2} v-h\left(\Delta x_{n} t_{k_{n}, N_{n}}+\Delta x_{n-1} t_{k_{n-1}, N_{n-1}}\right)\right)^{2},
$$

we obtain

$$
\left|\log L_{N}\left(n, v+a_{n-1} x+h \Delta x_{n-1} \sqrt{N} t_{k, N_{n-1}}\right)\right| \leq C N \exp (-N / C)
$$

Define

$$
x_{N}(v) \equiv \frac{\sqrt{N}}{a_{n-1}}\left(M(h)-\delta-v N^{-1 / 2}-a_{n}\left(2 \Delta x_{n}\left(\log 2-I\left(t_{k_{n}, N_{n}}\right)\right)\right)^{1 / 2}-h\left(\Delta x_{n} t_{k_{n}, N_{n}}+\Delta x_{n-1} t_{k_{n-1}, N_{n-1}}\right)\right) .
$$

Using the elementary bounds 


$$
1+x \leq e^{x} \leq 1+x+x^{2} \quad \text { for }|x|<1,
$$

and the bound (4.20), we obtain

$$
\begin{aligned}
& \mathbb{E}\left[\mathbb{1}_{\left\{X \leq x_{N}(v)\right\}} L_{N}\left(n, v+a_{n-1} X+h \Delta x_{n-1} \sqrt{N} t_{k_{n-1}, N_{n-1}}\right)\right] \\
& \quad \underset{N \uparrow+\infty}{=} \mathbb{P}\left\{X \leq x_{N}(v)\right\}+\mathbb{E}\left[\mathbb{1}_{\left\{X \leq x_{N}(v)\right\}} \log L_{N}\left(n, v+a_{n-1} X+h \Delta x_{n-1} \sqrt{N} t_{k_{n-1}, N_{n-1}}\right)\right]+\mathcal{O}(N \exp (-N / C)) .
\end{aligned}
$$

Given $k_{n-1} \in\left[1 ; N_{n-1}\right] \cap \mathbb{N}$, we have

$$
\begin{aligned}
\mathbb{E} & {\left[1_{\left\{X \leq x_{N}(v)\right\}} \exp \left(-\frac{1}{2 a_{n}^{2}} Y_{N}\left(h, y, v+a_{n-1} X+h \Delta x_{n-1} \sqrt{N} t_{k_{n-1}, N_{n-1}}, t_{k_{n}, N}, n\right)^{2}\right)\right] } \\
= & \frac{1}{\sqrt{2 \pi}} \int_{-\infty}^{x_{N}(v)} d x \exp \left[-\frac{x^{2}}{2}-\frac{1}{2 a_{n}^{2}}\left(u_{N, h}(y)-a_{n-1} x-h \sqrt{N}\left(\Delta x_{n} t_{k_{n}, N_{n}}+\Delta x_{n-1} t_{k_{n-1}, N_{n-1}}\right)-v\right)^{2}\right] \\
= & \exp \left(-\frac{1}{1-q_{n-2}} Y_{N}\left(h, y, v, t\left(k_{n}, k_{n-1}\right), n-1\right)^{2}\right) \\
& \times \frac{1}{\sqrt{2 \pi}} \int_{-\infty}^{x_{N}(v)} \exp \left[-\frac{a_{n}^{2}+a_{n-1}^{2}}{2 a_{n}^{2}}\left(x-\frac{a_{n-1}}{a_{n}^{2}+a_{n-1}^{2}} Y_{N}\left(h, y, v, t\left(k_{n}, k_{n-1}\right), n-1\right)\right)^{2}\right] d x .
\end{aligned}
$$

We claim that due to the strict inequalities (4.1), we have

$$
\frac{1}{\sqrt{2 \pi}} \int_{-\infty}^{x_{N}(v)} \exp \left[-\frac{a_{n}^{2}+a_{n-1}^{2}}{2 a_{n}^{2}}\left(x-\frac{a_{n-1}}{a_{n}^{2}+a_{n-1}^{2}} Y_{N}\left(h, y, v, t\left(k_{n}, k_{n-1}\right), n-1\right)\right)^{2}\right] d x \underset{N \uparrow+\infty}{\rightarrow} \frac{a_{n}}{\left(a_{n}^{2}+a_{n-1}^{2}\right)^{1 / 2}},
$$

uniformly in $v \in \mathbb{R}$ such that

$$
v \leq \sqrt{N}\left(M(h)+\delta^{\prime}-h\left(\Delta x_{n} t_{k_{n}, N_{n}}+\Delta x_{n-1} t_{k_{n-1}, N_{n-1}}\right)-\left(a_{n}^{2}+a_{n-1}^{2}\right) \mu(h)\right) \equiv v_{N}^{\max },
$$

where $0<\delta^{\prime}$ exists due to strict inequality (4.1), for $l=n$. Indeed, due to the standard bounds on Gaussian tails, to show (4.25) it is enough to check that

$$
\frac{a_{n-1}}{a_{n}^{2}+a_{n-1}^{2}} Y_{N}\left(h, y, v, t\left(k_{n}, k_{n-1}\right), n-1\right)+\delta \sqrt{N} \leq x_{N}(v),
$$

for $v$ satisfying (4.26). Due to (4.1) with $l=n$, there exists $\delta_{3}>0$ such that we have

$$
\left(2 \Delta x_{n}\left(\log 2-I\left(t_{k_{n}, N_{n}}\right)\right)\right)^{1 / 2} \leq \mu(h)-\delta_{3} .
$$

Choosing a small enough $\delta^{\prime}>0$, we have

$$
\begin{aligned}
x_{N}(v) & -\frac{a_{n-1}}{a_{n}^{2}+a_{n-1}^{2}} Y_{N}\left(h, y, v, t\left(k_{n}, k_{n-1}\right), n-1\right)+\delta \sqrt{N} \\
& =a_{n}^{2}\left(M(h)-v N^{-1 / 2}-h\left(\Delta x_{n} t_{k_{n}, N_{n}}+\Delta x_{n-1} t_{k_{n-1}, N_{n-1}}\right)\right) \\
& -\left(a_{n}^{2}+a_{n-1}^{2}\right)\left(a_{n}\left(2 \Delta x_{n}\left(\log 2-I\left(t_{k_{n}, N_{n}}\right)\right)\right)^{1 / 2}-\delta\right) \\
& \geq a_{n}^{2}\left(\left(a_{n}^{2}+a_{n-1}^{2}\right) \mu(h)-\delta^{\prime}\right)-\left(a_{n}^{2}+a_{n-1}^{2}\right)\left(a_{n}\left(2 \Delta x_{n}\left(\log 2-I\left(t_{k_{n}, N_{n}}\right)\right)\right)^{1 / 2}-\delta\right) \\
& 4.26 \\
& \geq a_{n}^{2}\left(\left(a_{n}^{2}+a_{n-1}^{2}\right) \mu(h)-\delta^{\prime}\right)-\left(a_{n}^{2}+a_{n-1}^{2}\right)\left(a_{n}^{2} \mu(h)-\delta\right)
\end{aligned}
$$




$$
\begin{aligned}
& =\left(a_{n}^{2}+a_{n-1}^{2}\right)\left(\delta_{3} a_{n}^{2}+\delta\right)-a_{n}^{2} \delta^{\prime} \\
& >0,
\end{aligned}
$$

which proves (4.27).

We claim that there exists $C>0$ such that uniformly in $k_{n-1} \in\left[1 ; N_{n-1}\right] \cap \mathbb{N}$ and in $v \in \mathbb{R}$ satisfying (4.26) we have

$$
\left(\begin{array}{c}
N_{n-1} \\
k_{n-1}
\end{array}\right) \mathbb{P}\left\{X \geq x_{N}(v)\right\} \leq \exp (-N / C) .
$$

Indeed, in view of (3.4) and due to the classical Gaussian tail asymptotics, to obtain (4.29) it is enough to show that

$$
N_{n-1}\left(\log 2-I\left(t_{k_{n-1}, N_{n-1}}\right)\right) \leq \frac{1}{2} x_{N}^{2}\left(v_{N}^{\max }\right) .
$$

Using (4.26) and (4.21), we obtain

$$
x_{N}\left(v_{N}^{\max }\right)=\frac{N^{1 / 2}}{a_{n-1}}\left(\left(a_{n}^{2}+a_{n-1}^{2}\right) \mu(h)-a_{n}\left(2 \Delta x_{n}\left(\log 2-I\left(t_{k_{n}, N_{n}}\right)\right)\right)^{1 / 2}+\delta^{\prime}-\delta\right) .
$$

If $n>2$, then due to strict inequality (4.1), for $l=n-2$, there exists $\delta^{\prime}>0$ such that we have

$$
\begin{aligned}
\left(a_{n}^{2}+a_{n-2}^{2}\right) \mu(h)-\delta^{\prime \prime} & >\left(\left(\log 2-I\left(t_{*}\left(\theta_{l, n}^{-1 / 2} h\right)\right)\right)\left(a_{n}^{2}+a_{n-2}^{2}\right)\left(\Delta x_{n}+\Delta x_{n-1}\right)\right)^{1 / 2} \\
& \geq\left(2 a_{n-1}^{2} \Delta x_{n-1}\left(\log 2-I\left(t_{k_{n-1}, N_{n-1}}\right)\right)\right)^{1 / 2}+\left(2 a_{n}^{2} \Delta x_{n}\left(\log 2-I\left(t_{k_{n}, N_{n}}\right)\right)\right)^{1 / 2},
\end{aligned}
$$

where the last inequality may be obtained as a consequence of Slepian's lemma. ${ }^{25}$ If $n=2$, then (4.32) follows directly from Slepian's lemma. Combining (4.31) and (4.32), we get (4.30). Note that (4.29), in particular, implies that

$$
\mathrm{P}\left\{X \geq x_{N}(v)\right\} \leq \exp (-N / C) .
$$

Given $k_{n-1} \in\left[1 ; N_{n-1}\right] \cap \mathbb{N}$, denote

$$
L_{N}\left(n-1, v, k_{n-1}\right) \equiv \mathbb{E}\left[L_{N}\left(n, v+a_{n-1} X+h \Delta x_{n-1} \sqrt{N} t_{k_{n-1}, N_{n-1}}\right)\right]\left(\begin{array}{l}
N_{n-1} \\
k_{n-1}
\end{array}\right) .
$$

Due to (4.33) and (4.23), we have

$$
\begin{aligned}
L_{N}\left(n-1, v, k_{n-1}\right)= & \mathbb{E}\left[\left(\mathbb{1}_{\left\{X \leq x_{N}(v)\right\}}+\mathbb{1}_{\left\{X>x_{N}(v)\right\}}\right) L_{N}\left(n, v+a_{n-1} X+h \Delta x_{n-1} \sqrt{N} t_{k_{n-1}, N_{n-1}}\right)\right]\left(\begin{array}{l}
N_{n-1} \\
k_{n-1}
\end{array}\right) \\
= & \left(1+\mathbb{E}\left[\mathbb{1}_{\left\{X \leq x_{N}(v)\right\}} L_{N}\left(n, v+a_{n-1} X+h \Delta x_{n-1} \sqrt{N} t_{k_{n-1}, N_{n-1}}\right)\right]\right. \\
& \left.+\mathcal{O}\left(\mathbb{P}\left\{X \geq x_{N}(v)\right\}+N \exp (-N / C)\right)\right)\left(\begin{array}{l}
N_{n-1} \\
k_{n-1}
\end{array}\right) .
\end{aligned}
$$

Using (4.29) and the standard bounds (4.17) and (4.22), we get

$$
\begin{aligned}
L_{N}\left(n-1, v, k_{n-1}\right)= & \exp \left\{\left(\begin{array}{c}
N_{n-1} \\
k_{n-1}
\end{array}\right) \mathbb{E}\left[\mathbb{1}_{\left\{X \leq x_{N}(v)\right\}} \log L_{N}\left(n, v+a_{n-1} X+h \Delta x_{n-1} \sqrt{N} t_{k_{n-1}, N_{n-1}}\right)\right]\right. \\
& +\mathcal{O}(N \exp (-N / C))\} .
\end{aligned}
$$

Applying (4.25), (4.24), and (4.11), for $l=n$, we obtain 


$$
\begin{aligned}
& \log L_{N}\left(n-1, v, k_{n-1}\right)=-\frac{A_{N}(h)}{\sqrt{2 \pi\left(a_{n}^{2}+a_{n-1}^{2}\right)}} \sum_{k_{n}=0}^{N_{n}}\left(\left(\begin{array}{c}
N_{n} \\
k_{n}
\end{array}\right)\left(\begin{array}{c}
N_{n-1} \\
k_{n-1}
\end{array}\right) \int_{\mathbb{R}}\left(1-e^{-\varphi(y)}\right)\right. \\
& \left.\times \exp \left[-\frac{1}{2\left(a_{n}^{2}+a_{n-1}^{2}\right)} Y_{N}\left(h, y, v, t_{k_{n}, k_{n-1}}, n-1\right)^{2}\right] d y\right)+\mathcal{O}(N \exp (-N / C)) .
\end{aligned}
$$

Finally, we arrive at

$$
\begin{aligned}
\log L_{N}(n-1, v)= & \sum_{k_{n-1}=0}^{N_{n-1}} \log L_{N}\left(n-1, v, k_{n-1}\right) \\
= & -\frac{A_{N}(h)}{\sqrt{2 \pi\left(a_{n}^{2}+a_{n-1}^{2}\right)}} \sum_{k_{n}=0}^{N_{n}} \sum_{k_{n-1}=0}^{N_{n-1}}\left(\left(\begin{array}{c}
N_{n} \\
k_{n}
\end{array}\right)\left(\begin{array}{c}
N_{n-1} \\
k_{n-1}
\end{array}\right)\right. \\
& \left.\times \int_{\mathbb{R}}\left(1-e^{-\varphi(y)}\right) \exp \left[-\frac{1}{2\left(a_{n}^{2}+a_{n-1}^{2}\right)} Y_{N}\left(h, y, v, t_{k_{n}, k_{n-1}}, n-1\right)^{2}\right] d y\right) \\
& +\mathcal{O}\left(N^{2} \exp (-N / C)\right) \\
= & -\frac{A_{N}(h)}{\sqrt{2 \pi\left(a_{n}^{2}+a_{n-1}^{2}\right)}} \sum_{k=0}^{N_{n}+N_{n-1}}\left(\left(\begin{array}{c}
N_{n}+N_{n-1} \\
k
\end{array}\right)\right. \\
& \left.\times \int_{\mathbb{R}}\left(1-e^{-\varphi(y)}\right) \exp \left[-\frac{1}{2\left(a_{n}^{2}+a_{n-1}^{2}\right)} Y_{N}\left(h, y, v, t_{k, N_{n}+N_{n-1}}, n-1\right)^{2}\right] d y\right) \\
& +\mathcal{O}\left(N^{2} \exp (-N / C)\right) .
\end{aligned}
$$

(4) Combining (4.9) and (4.11) for $l=1$, we obtain

$$
L_{\mathcal{E}_{N}(h)}(\varphi)=\exp \left(-\int_{\mathbb{R}}\left(1-e^{-\varphi(y)}\right) S_{N}(h, y) d y+o(1)\right)
$$

where $S_{N}(h, y)$ is given by (3.24). Invoking the proof of Theorem 1.1, we get that

$$
L_{\mathcal{E}_{N}(h)}(\varphi) \underset{N \uparrow+\infty}{\rightarrow} \exp \left(-\int_{\mathbb{R}}\left(1-e^{-\varphi(y)}\right) e^{-y} d y\right)=L_{\mathcal{P}\left(e^{-x}\right)}(\varphi)
$$

This establishes (4.2).

(5) The proof of (4.5) is very similar to the above proof of (4.2). The main difference is that (4.25) does not hold. Instead, if (4.4) holds for $l_{0}=n$, then we have

$$
\begin{aligned}
& \frac{1}{\sqrt{2 \pi}} \int_{-\infty}^{x_{N}(v)} \exp \left[-\frac{a_{n}^{2}+a_{n-1}^{2}}{2 a_{n}^{2}}\left(x-\frac{a_{n-1}}{a_{n}^{2}+a_{n-1}^{2}} Y_{N}\left(h, y, v, t\left(k_{n}, k_{n-1}\right), n-1\right)\right)^{2}\right] d x \\
& \underset{N \uparrow+\infty}{\rightarrow} \frac{a_{n}}{\left(a_{n}^{2}+a_{n-1}^{2}\right)^{1 / 2}} \mathbb{P}\left\{X<\frac{\sqrt{N}}{a_{n-1} \sqrt{a_{n}^{2}+a_{n-1}^{2}}}\left[M(h)-v N^{-1 / 2}-\left(1-x_{n-2}\right) h t_{*}\left(h \theta_{n, n}^{-1 / 2}\right)\right.\right. \\
& \left.\left.\quad-\left(a_{n}^{2}+a_{n-1}^{2}\right) \mu(h)\right]\right\}
\end{aligned}
$$

uniformly in 


$$
v \leq \sqrt{N}\left(M(h)-\left(1-x_{n-2}\right) h t_{*}\left(h \theta_{n, n}^{-1 / 2}\right)-\left(a_{n}^{2}+a_{n-1}^{2}\right) \mu(h)\right)-\delta^{\prime} .
$$

The subsequent applications of the recursion (4.10) to (4.35) give rise to the constant $K(h, \rho)$ $\in(0 ; 1)$ in $(4.5)$.

Proof of Theorem 1.2: The existence of the right hand side of (1.28) follows from the result of Bovier and Kurkova ${ }^{7}$ Theorem 1.5 (ii). It remains to show convergence (1.28) itself. We apply Proposition 4.1 to each coarse-grained block. Note that the assumption (4.1) of Proposition 4.1 is fulfilled due to the construction of the blocks, cf. (1.21) and (1.27). The result then follows from the result of Bovier and Kurkova ${ }^{7}$ Theorem 1.2.

The representation of the limiting ground state (1.29) is proven exactly as in Bovier and Kurkova $^{7}$ Theorem 1.5 (iii).

\section{B. Fluctuations of the partition function}

In this subsection we compute the limiting distribution of the GREM partition function under the scaling induced by (1.17). The analysis amounts to handling both the low and high temperature regimes. The low temperature regime is completely described by the behavior of the ground states which is summarized in Theorem 1.2. The high temperature regime is considered in Lemma 4.1 below.

Lemma 4.1: Assume $l(\beta, h)=0$. Then

$$
\exp \left[-N\left(\log 2+\log \operatorname{ch} \beta h+\frac{\beta^{2}}{2}\right)\right] \operatorname{ch}^{2 / 3}(\beta h) Z_{N}(\beta, h) \underset{N \uparrow+\infty}{\stackrel{w}{\rightarrow}} K(\beta, h),
$$

where $K(\beta, h)=1$, if $\beta \bar{\gamma}_{1}(h)<1$, and $K(\beta, h) \in(0 ; 1)$, if $\beta \bar{\gamma}_{1}(h)=1$.

Proof: We follow the strategy of Bovier and Kurkova ${ }^{7}$ Lemma 3.1. By the very construction of the coarse-graining algorithm (1.21), we have

$$
\begin{gathered}
\tilde{\theta}_{1, k} \leq \tilde{\theta}_{1, J_{1}}=\bar{\gamma}_{1}(h)^{2}, \quad k \in\left[1 ; J_{1}\right] \cap \mathbb{N}, \\
\tilde{\theta}_{1, k}<\tilde{\theta}_{1, J_{1}}, \quad k \in\left(J_{1} ; n\right] \cap \mathbb{N} .
\end{gathered}
$$

Assume $\beta \bar{\gamma}_{1}(h)<1$. Hence, due to (4.37), we have

$$
\beta \widetilde{\theta}_{1, k}^{1 / 2}<1, \quad k \in[1 ; n] \cap \mathbb{N} .
$$

Strict inequality (4.38) implies that there exists $\varepsilon>0$ such that, for all $k \in[1 ; n] \cap \mathbb{N}$,

$$
\left(\beta^{2}-\frac{1}{2}(\beta-\varepsilon)^{2}\right) q_{k}<x_{k}\left(\log 2-I\left(t_{*}\left(h\left(x_{k} / q_{k}\right)^{1 / 2}\right)\right)\right) .
$$

We have

$$
\mathbb{E}\left[Z_{N}(\beta, h)\right]=\sum_{k=0}^{N}\left(\begin{array}{l}
N \\
k
\end{array}\right) \exp \left(\beta h t_{k, N} N+\frac{\beta^{2} N}{2}\right) \equiv S_{N}(\beta, h) .
$$

Note that due to (3.4)

$$
S_{N}(\beta, h) \underset{N \uparrow+\infty}{\sim} \sum_{k=0}^{N} g_{N}\left(t_{k, N}\right) \exp \left(N f\left(t_{k, N}\right)\right)
$$

where

$$
f(t) \equiv \log 2-I(t)+\beta h t+\beta^{2} / 2,
$$




$$
g_{N}(t) \equiv\left(\frac{2}{\pi N\left(1-t^{2}\right)}\right)^{1 / 2}
$$

A straightforward computation gives

$$
\begin{gathered}
f^{\prime}\left(t_{0}\right)=\beta h-\tanh ^{-1}\left(t_{0}(\beta, h)\right)=0, \\
f^{\prime \prime}\left(t_{0}\right)=-\left(1-t_{0}^{2}\right)^{-1}=-\operatorname{ch}^{2}(\beta h), \\
g_{N}\left(t_{0}\right)=\left(\frac{2}{\pi N\left(1-t^{2}\right)}\right)^{1 / 2}=\left(\frac{2}{\pi N}\right)^{1 / 2} \operatorname{ch}(\beta h) .
\end{gathered}
$$

The asymptotic Laplace method then yields

$$
S_{N}(\beta, h) \underset{N \uparrow+\infty}{\sim} \operatorname{ch}^{-2 / 3}(\beta h) \exp \left[N\left(\log 2+\log \operatorname{ch} \beta h+\frac{\beta^{2}}{2}\right)\right] .
$$

For $p \leq q$, define

$$
\operatorname{GREM}_{N}^{(p, q)}\left(\sigma^{(1)}, \ldots, \sigma^{(q)}\right) \equiv \sum_{k=p}^{q} a_{k} X\left(\sigma^{(1)}, \ldots, \sigma^{(k)}\right)
$$

Consider the event

$$
E_{N}(\sigma) \equiv\left\{\operatorname{GREM}_{N}^{(1, k)}\left(\sigma^{(1)}, \ldots, \sigma^{(k)}\right)<(\beta+\varepsilon) q_{k} \sqrt{N} \text { for all } k \in[1 ; n] \cap \mathbb{N}\right\} .
$$

Define the truncated partition function as

$$
Z_{N}^{(\mathrm{T})}(\beta, h) \equiv \sum_{\sigma \in \Sigma_{N}} \mathbb{1}_{E_{N}(\sigma)} \exp \left[\beta \sqrt{N} X_{N}(h, \sigma)\right]
$$

The truncation (4.43) is mild enough in the following sense:

$$
\begin{aligned}
\mathbb{E}\left[Z_{N}^{(\mathrm{T})}(\beta)\right] & =S_{N}(\beta, h) \mathbb{P}\left\{\operatorname{GREM}_{N}^{(1, k)}\left(\sigma^{(1)}, \ldots, \sigma^{(k)}\right)<\varepsilon q_{k} \sqrt{N}, \quad \text { for all } k \in[1 ; n] \cap \mathbb{N}\right\} \\
& \sim \underset{N \uparrow+\infty}{\sim} \mathbb{E}\left[Z_{N}(\beta, h)\right] .
\end{aligned}
$$

We write

$$
\frac{Z_{N}(\beta)}{\mathbb{E}\left[Z_{N}(\beta)\right]}=\frac{Z_{N}^{(\mathrm{T})}(\beta)}{\mathbb{E}\left[Z_{N}^{(\mathrm{T})}(\beta)\right]} \times \frac{\mathbb{E}\left[Z_{N}^{(\mathrm{T})}(\beta)\right]}{\mathbb{E}\left[Z_{N}(\beta)\right]}+\frac{Z_{N}(\beta)-Z_{N}^{(\mathrm{T})}(\beta)}{\mathbb{E}\left[Z_{N}(\beta)\right]} \equiv(\mathrm{I}) \times(\mathrm{II})+(\mathrm{III}) .
$$

Due to (4.44), we get

$$
\text { (II) } \underset{N \uparrow+\infty}{\sim} 1, \quad(\mathrm{III}) \underset{N \uparrow+\infty}{\stackrel{L^{1}}{\longrightarrow}} 0 .
$$

To estimate (I), we fix any $\delta>0$ and use the Chebyshev inequality

$$
\mathbb{P}\{|(\mathrm{I})-1|>\delta\} \leq\left(\delta \mathbb{E}\left[Z_{N}^{(\mathrm{T})}(\beta)\right]\right)^{-2} \operatorname{Var}\left[Z_{N}^{(\mathrm{T})}(\beta)\right] .
$$

Expanding the squares, we have 


$$
\begin{aligned}
\operatorname{Var}\left[Z_{N}^{(\mathrm{T})}(\beta)\right]= & \mathbb{E}\left[Z_{N}^{(\mathrm{T})}(\beta)^{2}\right]-\mathbb{E}\left[Z_{N}^{(\mathrm{T})}(\beta)\right]^{2} \\
= & \sum_{p=1}^{n} \sum_{\sigma^{(1)} \| \ldots . . \sigma^{(k)} \in \Sigma_{x_{k} N}} \mathbb{E}\left[\operatorname { e x p } \left\{2 \beta \sqrt { N } \left(\operatorname{GREM}_{N}^{(1, p)}\left(\sigma^{(1)}, \ldots, \sigma^{(p)}\right)\right.\right.\right. \\
& \left.\left.+2 \beta h x_{p} m_{x_{p} N}\left(\sigma^{(1)}, \ldots, \sigma^{(p)}\right) \sqrt{N}\right)\right\} \\
& \times \sum_{\sigma^{(p+1)}\|\ldots\| \sigma^{(n)},} \exp \left\{\beta \sqrt { N } \left(\operatorname{GREM}_{N}^{(p+1, n)}\left(\sigma^{(1)}, \ldots, \sigma^{(n)}\right)\right.\right. \\
& +\operatorname{GREM}_{\tau^{(p+1)}\|\ldots\| \tau^{(n)} \in \Sigma_{\left(1-x_{p}\right) N},}^{(p+1, n)}\left(\tau^{(1)}, \ldots, \tau^{(n)}\right)+h\left(1-x_{p}\right) \sqrt{N}\left(m_{\left(1-x_{p}\right) N}\left(\sigma^{(p+1)}\|\ldots\| \sigma^{(n)}\right)\right. \\
& \left.\left.\left.\left.\left.\left.+m_{\left(1-x_{p}\right) N}\left(\tau^{(p+1)}\|\ldots\| \tau^{(n)}\right)\right)\right)\right\}\right]_{E_{N}\left(\sigma^{(1)}\|\ldots . .\| \sigma^{(n)}\right)}\right]_{E_{N}\left(\tau^{(1)} \| \ldots . . \tau^{(n)}\right)}\right] .
\end{aligned}
$$

Hence, due to the independence, we arrive at

$$
\begin{aligned}
& \operatorname{Var}\left[Z_{N}^{(\mathrm{T})}(\beta)\right] \leq \sum_{p=1}^{n} \sum_{k=0}^{x_{k} N}\left(\begin{array}{l}
N \\
k
\end{array}\right) \mathbb{E}\left[\operatorname { e x p } \left\{2 \beta \sqrt { N } \left(\operatorname{GREM}_{N}^{(1, p)}\left(\sigma^{(1)}, \ldots, \sigma^{(p)}\right)\right.\right.\right. \\
& \left.\left.\left.+h x_{p} t_{k, N} \sqrt{N}\right)\right\} \mathbb{1}_{\left\{\operatorname{GREM}_{N}^{(1, p)}\left(\sigma^{(1)}, \ldots, \sigma^{(p)}\right)<(\beta+\varepsilon) q_{p} \sqrt{N}\right\}}\right] \\
& \times\left(\sum _ { k = 0 } ^ { ( 1 - x _ { p } ) N } ( \begin{array} { c } 
{ ( 1 - x _ { p } ) N } \\
{ k }
\end{array} ) \mathbb { E } \left[\operatorname { e x p } \left(\beta \sqrt { N } \left(\operatorname{GREM}_{N}^{(p+1, n)}\left(\sigma^{(1)}, \ldots, \sigma^{(n)}\right)\right.\right.\right.\right. \\
& \left.\left.\left.+h\left(1-x_{p}\right) t_{k,\left(1-x_{p}\right) N} \sqrt{N}\right)\right]\right)^{2} \text {. }
\end{aligned}
$$

Assume that $X$ is a standard Gaussian random variable. Using the standard Gaussian tail bounds, we have

$$
\begin{aligned}
& \mathbb{E}\left[\exp \left(2 \beta \sqrt{N}\left(\operatorname{GREM}_{N}^{(1, p)}\left(\sigma^{(1)}, \ldots, \sigma^{(p)}\right)+h x_{p} t_{k, N} \sqrt{N}\right)\right) \mathbb{1}_{\left\{\operatorname{GREM}_{N}^{(1, p)}\left(\sigma^{(1)}, \ldots, \sigma^{(p)}\right)<(\beta+\varepsilon) q_{p} \sqrt{N}\right\}}\right] \\
& \quad=\exp \left\{N\left(2 \beta^{2} q_{p}+\beta h t_{k, N}\right)\right\} \mathrm{P}\left\{X \geq(\beta-\varepsilon) \sqrt{q_{p} N}\right\} \\
& \quad \underset{N \uparrow+\infty}{\leq} C \exp \left\{N\left(2 \beta^{2} q_{p}+\beta h t_{k, N}-\frac{1}{2}(\beta-\varepsilon)^{2} q_{p}\right)\right\} .
\end{aligned}
$$

Similarly to (4.41), using (3.4) and (4.48), we have

$$
\begin{aligned}
& \sum_{k=0}^{x_{k} N}\left(\begin{array}{c}
N \\
k
\end{array}\right) \mathbb{E}\left[\exp \left(2 \beta \sqrt{N}\left(\operatorname{GREM}_{N}^{(1, p)}\left(\sigma^{(1)}, \ldots, \sigma^{(p)}\right)+h x_{p} t_{k, N} \sqrt{N}\right)\right) 1_{\left\{\operatorname{GREM}_{N}^{(1, p)}\left(\sigma^{(1)}, \ldots, \sigma^{(p)}\right)<(\beta+\varepsilon) q_{p} \sqrt{N}\right\}}\right] \\
& \underset{N \uparrow+\infty}{\leq} C \sum_{k=0}^{x_{k} N} \exp \left\{N\left(x_{p}\left(\log 2-I\left(t_{k, x_{p} N}\right)\right)+2 \beta^{2} q_{p}+2 \beta h x_{p} t_{k, x_{p} N}-\frac{1}{2}(\beta-\varepsilon)^{2} q_{p}\right)\right\} \\
& \quad \equiv P_{N}(p) .
\end{aligned}
$$

Using (3.4), we also obtain 


$$
\begin{aligned}
& \sum_{k=0}^{\left(1-x_{p}\right) N}\left(\begin{array}{c}
\left(1-x_{p}\right) N \\
k
\end{array}\right) \mathbb{E}\left[\exp \left(\beta \sqrt{N}\left(\operatorname{GREM}_{N}^{(p+1, n)}\left(\sigma^{(1)}, \ldots, \sigma^{(n)}\right)+h\left(1-x_{p}\right) \sqrt{N} t_{k,\left(1-x_{p}\right) N}\right)\right]\right. \\
& \underset{N \uparrow+\infty}{\leq} C \sum_{k=0}^{\left(1-x_{p}\right) N} \exp \left\{N\left(\left(1-x_{p}\right)\left(\log 2-I\left(t_{k,\left(1-x_{p}\right) N}\right)\right)+\frac{1}{2}\left(1-q_{p}\right) \beta^{2}+\beta h\left(1-x_{p}\right) t_{k,\left(1-x_{p}\right) N}\right)\right\} \\
& \equiv \widetilde{P}_{N}(p) .
\end{aligned}
$$

Combining (4.47), (4.49), and (4.50), we get

$$
\operatorname{Var}\left[Z_{N}^{(\mathrm{T})}(\beta)\right] \underset{N \uparrow+\infty}{\leq} \sum_{p=1}^{N} P_{N}(p) \widetilde{P}_{N}^{2}(p) .
$$

For any $p \in[1 ; n] \cap \mathbb{N}$, we have the following factorization:

$$
\begin{aligned}
\mathbb{E}\left[Z_{N}^{(\mathrm{T})}(\beta)\right]= & \sum_{k=0}^{x_{p} N}\left(\begin{array}{c}
x_{p} N \\
k
\end{array}\right) \mathbb{E}\left[\exp \left(\beta \sqrt{N}\left(\operatorname{GREM}_{N}^{(1, p)}\left(\sigma^{(1)}, \ldots, \sigma^{(p)}\right)+h x_{p} t_{k, x_{p} N} \sqrt{N}\right)\right)\right. \\
& \times \sum_{k=0}^{\left(1-x_{p}\right) N}\left(\begin{array}{c}
\left(1-x_{p}\right) N \\
k
\end{array}\right) \exp \left(\beta \sqrt { N } \left(\operatorname{GREM}_{N}^{(p+1, n)}\left(\sigma^{(1)}, \ldots, \sigma^{(n)}\right)\right.\right. \\
& \left.\left.\left.+h\left(1-x_{p}\right) N t_{k,\left(1-x_{p}\right) N} \sqrt{N}\right)\right) 1_{E_{N}\left(\sigma^{(1)}\|\ldots\| \sigma^{(n)}\right)}\right]
\end{aligned}
$$

Hence, again similarly to (4.41), we obtain

$$
\begin{aligned}
\mathbb{E}\left[Z_{N}^{(\mathrm{T})}(\beta)\right] \underset{N \uparrow+\infty}{\sim} & C \sum_{k=0}^{x_{p} N} \exp \left\{N\left(x_{p}\left(\log 2-I\left(t_{k, x_{p} N}\right)\right)+\frac{1}{2} q_{p} \beta^{2}+\beta h x_{p} t_{k, x_{p} N}\right)\right\} \\
& \times \sum_{k=0}^{\left(1-x_{p}\right) N} \exp \left\{N\left(\left(1-x_{p}\right)\left(\log 2-I\left(t_{k,\left(1-x_{p}\right) N}\right)\right)+\frac{1}{2}\left(1-q_{p}\right) \beta^{2}+\beta h\left(1-x_{p}\right) t_{k,\left(1-x_{p}\right) N}\right)\right\} \\
\equiv & Q_{N}(p) \widetilde{P}_{N}(p) .
\end{aligned}
$$

Denote

$$
R_{N}(p) \equiv q_{p} \beta^{2}+2 x_{p} \max _{t \in[-1 ; 1]}\{\log 2-I(t)+\beta h t\} .
$$

We observe that similar to (4.42) we have

$$
\frac{Q_{N}^{2}(p)}{\exp (N R(p))_{N \uparrow+\infty}} \sim \underset{ }{\sim} \text {. }
$$

Combining (4.51), (4.53), (4.54), and (3.14), we get

$$
\begin{aligned}
& \text { (4.45) } \underset{N \uparrow+\infty}{\leq} C \sum_{p=1}^{n} \frac{P_{N}(p)}{Q_{N}^{2}(p)}=C \sum_{p=1}^{n} \frac{P_{N}(p) / \exp (N R(p))}{Q_{N}^{2}(p) / \exp (N R(p))} \\
& \underset{N \uparrow+\infty}{\leq} \sum_{p=1}^{n} \frac{P_{N}(p)}{\exp (N R(p))} \underset{N \uparrow+\infty}{\leq} C \sum_{p=1}^{n} \exp \left\{N\left(\left(\beta^{2}-\frac{1}{2}(\beta-\varepsilon)^{2}\right) q_{p}-\left(\log 2-I\left(t_{0}\right)\right) x_{p}\right)\right\} \\
& =C \sum_{p=1}^{n} \exp \left\{N\left(\left(\beta^{2}-\frac{1}{2}(\beta-\varepsilon)^{2}\right) q_{p}-\left(\log 2-I\left(t_{*}\left(h\left(x_{p} / q_{p}\right)^{1 / 2}\right)\right)\right) x_{p}\right)\right\}
\end{aligned}
$$




$$
\underset{N \uparrow+\infty}{\longrightarrow} 0
$$

where the convergence to zero in the last line is assured by the choice of $\varepsilon$ in (4.39). Finally, combining (4.45) and (4.55), we get

$$
\text { (I) } \underset{N \uparrow+\infty}{\stackrel{P}{\longrightarrow}} 1 .
$$

This finishes the proof of (4.36) in the case $\beta \bar{\gamma}_{1}(h)<1$.

The case $\beta \bar{\gamma}_{1}(h)=1$ is a little bit more tedious and uses the information about the low temperature regime obtained in Theorem 1.2 in the spirit of the proof of Bovier and Kurkova ${ }^{7}$ Lemma 3.1. The lemma follows.

Proof of Theorem 1.3: The proof is verbatim the one of Bovier and Kurkova ${ }^{7}$ Theorem 1.7, where the analysis of the high temperature regime Bovier and Kurkova ${ }^{7}$ Lemma 3.1 is substituted by Lemma 4.1. The low temperature regime is governed by the fluctuations of the ground state which are summarized in Theorem 1.2.

\section{Formula for the free energy of the GREM}

Proof of Theorem 1.4: The $L^{1}$ convergence follows immediately from Theorem 1.3. Almost sure convergence is a standard consequence of Gaussian measure concentration, e.g., Ledoux ${ }^{24}$ (2.35), and the Borell-Cantelli lemma.

\section{ACKNOWLEDGMENTS}

A.K. gratefully acknowledges financial support of the DFG Research Training Group "Stochastic Processes and Probabilistic Analysis" and of the Helmholz-Gemeinschaft.

${ }^{1}$ F. Guerra, Commun. Math. Phys. 233, 1 (2003).

${ }^{2}$ M. Aizenman, R. Sims, and S. L. Starr, Phys. Rev. B 68, 214403 (2003).

${ }^{3}$ M. Aizenman, R. Sims, and S. L. Starr, Prospects in Mathematical Physics, Contemporary Mathematics Vol. 437 (American Mathematical Society, Providence, RI, 2007), pp. 1-30; e-print arXiv:math-ph/0607060.

${ }^{4}$ M. Talagrand. Ann. Math. 163, 221 (2006).

${ }^{5}$ B. Derrida, J. Phys. (France) 46, 401 (1985).

${ }^{6}$ A. Bovier, Statistical Mechanics of Disordered Systems, Cambridge Series in Statistical and Probabilistic Mathematics (Cambridge University Press, Cambridge, 2006).

${ }^{7}$ A. Bovier and I. Kurkova, Ann. I.H.P. Probab. Stat. 40, 439 (2004).

${ }^{8}$ A. Bovier and I. Kurkova, Ann. I.H.P. Probab. Stat. 40, 481 (2004).

${ }^{9}$ A. Bovier and I. Kurkova, available at http://www.wias-berlin.de/people/bovier/files/bk4-good.pdf.

${ }^{10}$ G. Ben Arous, L. V. Bogachev, and S. A. Molchanov, Probab. Theory Relat. Fields 132, 579 (2005).

${ }^{11}$ B. Derrida and E. Gardner, J. Phys. C 19, 5783 (1986).

${ }^{12}$ A. Bovier and I. Kurkova, Spin Glasses, Lecture Notes in Mathematics Vol. 1900 (Springer, Berlin, 2007), pp. 81-115.

${ }^{13}$ N. K. Jana and B. V. Rao, e-print arXiv:math/0612836v1.

${ }^{14}$ N. K. Jana, "Contributions to Random Energy Models," Ph.D. thesis, Indian Statistical Institute, 2007.

${ }^{15}$ A. Bovier, I. Kurkova, and M. Löwe, Ann. Probab. 30, 605 (2002).

${ }^{16}$ D. Ruelle, Commun. Math. Phys. 108, 225 (1987).

${ }^{17}$ F. Guerra and F. L. Toninelli, Markov Processes Relat. Fields 9, 195 (2003).

${ }^{18}$ P. Contucci, M. D. Esposti, C. Giardinà, and S. Graffi, Commun. Math. Phys. 236, 55 (2003).

${ }^{19}$ A. Bovier and A. Klimovsky, e-print arXiv:0802.3467v1.

${ }^{20}$ B. Derrida, Phys. Rev. Lett. 45, 79 (1980).

${ }^{21}$ T. Eisele, Commun. Math. Phys. 90, 125 (1983).

${ }^{22}$ E. Olivieri and P. Picco, Commun. Math. Phys. 96, 125 (1984).

${ }^{23}$ T. C. Dorlas and J. R. Wedagedera, Int. J. Mod. Phys. B 15, 1 (2001).

${ }^{24}$ M. Ledoux, The Concentration of Measure Phenomenon, Mathematical Surveys and Monographs Vol. 89 (American Mathematical Society, Providence, RI, 2001).

${ }^{25}$ D. Slepian, Bell Syst. Tech. J. 41, 463 (1962). 